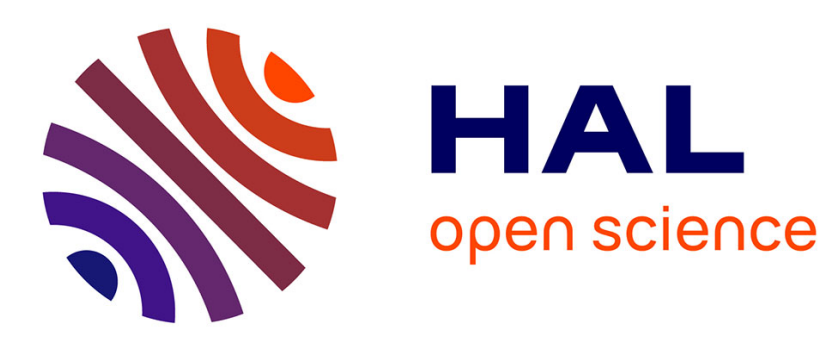

\title{
Quotients and invariants of AS-sets equipped with a finite group action
}

\author{
Fabien Priziac
}

\section{To cite this version:}

Fabien Priziac. Quotients and invariants of AS-sets equipped with a finite group action. Mathematische Annalen, 2020, 10.1007/s00208-020-01994-7 . hal-02063373v3

\section{HAL Id: hal-02063373 https://hal.science/hal-02063373v3}

Submitted on 29 Apr 2020

HAL is a multi-disciplinary open access archive for the deposit and dissemination of scientific research documents, whether they are published or not. The documents may come from teaching and research institutions in France or abroad, or from public or private research centers.
L'archive ouverte pluridisciplinaire HAL, est destinée au dépôt et à la diffusion de documents scientifiques de niveau recherche, publiés ou non, émanant des établissements d'enseignement et de recherche français ou étrangers, des laboratoires publics ou privés. 


\title{
Quotients and invariants of $\mathcal{A S}$-sets equipped with a finite group action
}

\author{
Fabien Priziac
}

\begin{abstract}
Using the geometric quotient of a real algebraic set by the action of a finite group $G$, we construct invariants of $G$-affine real algebraic varieties with respect to equivariant homeomorphisms with algebraic graph, including additive invariants with values in $\mathbb{Z}$. The construction requires to consider the wider category of $\mathcal{A S}$-sets.
\end{abstract}

\section{Contents}

1 Introduction 2

2 Quotient of an arc-symmetric set by a free finite group action 4

2.1 Quotient of a real algebraic set by a finite group action . . . . . . . . . . . 4

2.2 Quotient of a semialgebraic set by a polynomial finite group action . . . . . . 7

2.3 Construction of the quotient of an arc-symmetric set by a free finite group action 9

2.4 Functoriality and uniqueness of the quotient of a free $G$ - $\mathcal{A S}$-set . . . . . . . . 11

3 Equivariant homologies and cohomologies $\quad 13$

3.1 Equivariant singular homology and cohomology . . . . . . . . . . . . . . 13

3.2 A real algebraic model for the total space $E_{G} \ldots \ldots \ldots \ldots \ldots$

3.3 Equivariant homology with closed supports and equivariant cohomology with compact supports . . . . . . . . . . . . . . . . . . . 18

4 The $G$-Nash constructible filtrations $\quad 21$

4.1 The homological $G$-Nash constructible filtration . . . . . . . . . . . . . . 21

4.2 The induced $G$-weight spectral sequence . . . . . . . . . . . . . 25

4.3 The $G$-virtual Betti numbers and the $G$-virtual Poincaré series . . . . . . . . 27

4.4 The dual $G$-Nash constructible filtration and the finiteness of the $G$-virtual Poincaré series . . . . . . . . . . . . . . . . . 33

5 Other properties of the $G$-virtual Poincaré series and applications

Keywords : real algebraic sets, $\mathcal{A S}$-sets, group action, real geometric quotient, equivariant (co)homology, weight filtration, additive invariants.

2010 Mathematics Subject Classification : 14P05, 14P10, 14P20, 14L24, 57S17. 


\section{Introduction}

In [15], C. McCrory and A. Parusiński showed the existence of additive invariants, with respect to biregular isomorphisms, of affine real algebraic varieties with values in $\mathbb{Z}$. These invariants, denoted by $\beta_{q}$, coincide with the classical Betti numbers on compact nonsingular varieties and are called the virtual Betti numbers. In [16], C. McCrory and A. Parusiński proved that the virtual Betti numbers are actually invariant under homeomorphisms with algebraic graph. To show this, they considered a wider and more flexible class of $\mathcal{A S}$-sets (see below definition $2.15)$ and associated to any $\mathcal{A S}$-set $X$ a filtered chain complex $\mathcal{N} C_{*}(X)$, called the Nash constructible filtration on closed semialgebraic $\mathbb{F}_{2}$-chains. From the induced spectral sequence, one can recover the virtual Betti numbers $\beta_{q}$. Since the Nash-constructible filtration is invariant under homeomorphisms with $\mathcal{A S}$-graph, so are the virtual Betti numbers. Notice that the Nash constructible filtration also induces a weight filtration on homology with closed supports and coefficients in $\mathbb{F}_{2}$, analogous to the weight filtration of complex algebraic varieties.

Let $G$ be a finite group. In this paper, we consider affine real algebraic varieties equipped with an action of $G$ by homeomorphisms with algebraic graph. We construct additive invariants $\beta_{q}(\cdot ; G)$ of such objects with values in $\mathbb{Z}$, which coincide with the Betti numbers of classical equivariant (co)homology (the one defined using the Borel construction) on compact nonsingular varieties : we call them the $G$-virtual Betti numbers (they are different from the equivariant virtual Betti numbers of [10]). To achieve this goal, we define an equivariant analog of the Nash constructible filtration, the $G$-Nash constructible filtration, and extract the $G$-virtual Betti numbers from the induced spectral sequence. By construction, the $G$-Nash constructible filtration is an invariant with respect to equivariant homeomorphisms with $\mathcal{A S}$ (e.g. algebraic) graph. As a consequence, so are the $G$-virtual Betti numbers.

The construction of the $G$-Nash constructible filtration is inspired by the definition of classical equivariant (co)homology and goes as follows. Let $X$ be a $G$-affine real algebraic variety. The equivariant singular homology $H_{*}^{G}(X)$ of $X$ is the singular homology of the (topological) Borel quotient of $X$ by $G$, that is the quotient of $X \times E_{G}$ by $G$, where $E_{G}$ is any contractible topological space equipped with a free action of $G$. We can find a "real algebraic" model for $E_{G}$, namely the infinite real Stiefel manifold $V_{|G|}\left(\mathbb{R}^{\infty}\right)=\lim _{\rightarrow \in \mathbb{N}} V_{|G|}\left(\mathbb{R}^{k}\right)$, so that $H_{*}^{G}(X)$ is the inductive limit of the homology of the quotients $X \times \overrightarrow{V_{|G|}}\left(\mathbb{R}^{k}\right) / G$. A key point is that each of these quotients has a well-defined $\mathcal{A S}$-structure (notice that, in general, the quotient of a real algebraic set by a group action is just a semialgebraic set). This is based on a statement due to G. Fichou ([10]) which says that the quotient of a compact real algebraic set by a free action of a finite group is a compact $\mathcal{A S}$-set. Now, the Nash constructible filtration of each quotient can be defined and the $G$-Nash constructible filtration $\mathcal{N} C_{*}(X ; G)$ of $X$ is the inductive limit $\lim _{k \in \mathbb{N}} \mathcal{N} C_{*}\left(X \times V_{|G|}\left(\mathbb{R}^{k}\right) / G\right)$. Actually, we perform all this construction in

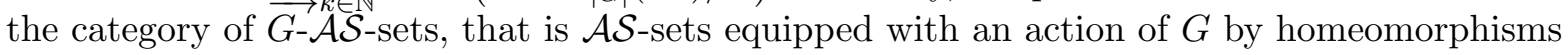
with $\mathcal{A S}$-graph.

For compact $G$-affine real algebraic varieties, the $G$-Nash constructible filtration induces a filtration on $H_{*}^{G}$ that we call the $G$-weight filtration in analogy with the non-equivariant case $([16])$. It is different from the equivariant weight filtration of $[20]$ (which was based on a different equivariant homology).

In a subsequent work, we plan to use the $G$-virtual Betti numbers for the classification of 
real analytic germs. More precisely, denote by $\beta(\cdot ; G)$ the generating function of the $G$-virtual Betti numbers : we call it the $G$-virtual Poincaré series. Since the $G$-virtual Poincaré series is an invariant with respect to equivariant homeomorphisms with $\mathcal{A S}$-graph and because it verifies some multiplicativity property (proposition 5.1), it should prove useful to define invariants of some equivalence of equivariant semialgebraic and analytic real germs, and to complete the study initiated in [21] and [22].

The structure of the paper is as follows.

In section 2.1, we review the definition and properties of the geometric quotient of a real algebraic set by a finite group action via polynomial maps. We focus on the properties that we need in the rest of the paper, such as functoriality or regularity.

In section 2, we give a well-defined (up to homeomorphism with $\mathcal{A S}$-graph) and functorial $\mathcal{A S}$-structure on the quotient of a free $G-\mathcal{A S}$-set, that is a $G-\mathcal{A S}$-set such that the action of $G$ on the compact arc-symmetric closure of an equivariant orthogonal embedding (proposition 2.19 ) is free. This uses the key proposition 2.20.

In section 3 , we give the definition and the basic properties of the equivariant homologies and cohomologies with respect to which are constructed the equivariant $\mathcal{A S}$-invariants of section 4 . The first paragraph is dedicated to the equivariant singular homology and cohomology, defined using the Borel construction. We show in particular that they can be computed using the Stiefel manifolds and the real geometric quotient (proposition 3.5 and corollary 3.6). In the second part, we define the equivariant homology with closed supports (as well as the equivariant cohomology with compact supports), as the homology of the inductive limit of the semialgebraic chain complexes with closed supports ([16] Appendix) of the quotients $X \times V_{|G|}\left(\mathbb{R}^{k}\right) / G, k \in \mathbb{N}$. The two equivariant (co)homologies, singular and with closed/compact supports, coincide on compact $G$ - $\mathcal{A S}$-sets (lemma 3.9 ).

In section 4, we construct the $G$-Nash constructible filtration : if $X$ is $G$ - $\mathcal{A S}$-set, it is the inductive limit of the Nash constructible filtrations of the $\mathcal{A S}$-quotients $X \times V_{|G|}\left(\mathbb{R}^{k}\right) / G$. We then prove three essential properties of the $G$-Nash constructible filtration : it is functorial with respect to equivariant proper continuous maps with $\mathcal{A S}$ graph (theorem 4.3), it is additive with respect to equivariant closed inclusions in terms of a short exact sequence (theorem 4.4) and the lines of the induced spectral sequence are bounded (theorem 4.11). We then define the $G$-virtual Betti numbers (theorem 4.12). At the end of this section, we also define the cohomological counterpart of the $G$-Nash constructible filtration (definition 4.20) and give its properties. In particular, we notice the finiteness of the $G$-virtual Poincaré series : it can be written as a rational fraction whose denominator depends only on the group $G$. Therefore, the information encoded by the $G$-virtual Poincaré series can actually be encoded in a polynomial with integer coefficients.

The final section of this paper is dedicated to some further properties of the $G$-virtual Poincaré series, including the statement that should be useful for the classification of real analytic germs (proposition 5.1). The other proposition 5.3 gives the behaviour of the $G$ virtual Poincaré series on free $G-\mathcal{A S}$-sets and on $\mathcal{A S}$-sets equipped with a trivial action of $G$.

Throughout this paper, $G$ will always denote a finite group and $\mathbb{F}_{2}$ will denote the twoelements field. 
Acknowledgements. The author wishes to thank J.-B. Campesato, G. Fichou and A. Parusiński for useful discussions and comments.

\section{Quotient of an arc-symmetric set by a free finite group action}

\subsection{Quotient of a real algebraic set by a finite group action}

Let $G$ be a finite group of order $N \in \mathbb{N} \backslash\{0\}$.

In this paragraph, we will consider a real algebraic set $X \subset \mathbb{R}^{d}$ on which $G$ acts via polynomial maps $\alpha_{g}: X \rightarrow X, g \in G$ : we will say that $X$ is a $G$-real algebraic set. We are going to recall the construction of the semialgebraic geometric quotient of $X$ by $G$ (see also for instance [24] or [18]).

Denote by $\mathcal{P}(X):=\mathbb{R}\left[x_{1}, \ldots, x_{d}\right] / I(X)$ the $\mathbb{R}$-algebra of polynomial functions on $X$. The action of $G$ on $X$ induces an action of $G$ on $\mathcal{P}(X)$, defined by

$$
g \cdot f:=f \circ \alpha_{g}^{-1}
$$

if $f \in \mathcal{P}(X)$ and $g \in G$. Since $\mathcal{P}(X)$ is a finitely generated $\mathbb{R}$-algebra and $G$ is finite, by a theorem of E. Noether ([17], see also, for instance, [27] Algebraic Appendix, section 4), the subalgebra $\mathcal{P}(X)^{G}$ of invariant polynomial functions on $X$ is a finitely generated $\mathbb{R}$-algebra as well : there exist invariant polynomial functions $p_{1}, \ldots, p_{m}$ on $X$ which generate $\mathcal{P}(X)^{G}$ as an $\mathbb{R}$-algebra.

Now, consider the complexification $X_{\mathbb{C}} \subset \mathbb{C}^{d}$ of $X$ : it is, by definition, the Zariski closure of $X$ considered as a subset of $\mathbb{C}^{d}$ (see [1] II.2). Notice that the coordinate ring $\mathbb{C}\left[X_{\mathbb{C}}\right]$ of $X_{\mathbb{C}}$ is the tensor product $\mathcal{P}(X) \otimes_{\mathbb{R}} \mathbb{C}$. We extend linearly the action of $G$ on $\mathcal{P}(X)$ into an action on $\mathbb{C}\left[X_{\mathbb{C}}\right]$, which corresponds (via the contrafunctorial equivalence between the category of complex algebraic sets and the category of reduced finitely generated $\mathbb{C}$-algebras, given by the Nullstellensatz) to an action of $G$ on $X_{\mathbb{C}}$ (the "complexified" action being given by the same polynomials with real coefficients as for the action of $G$ on $X$ ).

Since the action of $G$ on $\mathbb{C}\left[X_{\mathbb{C}}\right]=\mathcal{P}(X) \otimes_{\mathbb{R}} \mathbb{C}$ is induced by linear extension, we have $\mathbb{C}\left[X_{\mathbb{C}}\right]^{G}=\mathcal{P}(X)^{G} \otimes_{\mathbb{R}} \mathbb{C}$ and the $\mathbb{C}$-algebra $\mathbb{C}\left[X_{\mathbb{C}}\right]^{G}$ is then generated (as a $\mathbb{C}$-algebra) by the invariant real polynomial functions $p_{1}, \ldots, p_{m}$ (considered as functions on $X_{\mathbb{C}}$ ).

Therefore, the reduced finitely generated $\mathbb{C}$-algebra $\mathbb{C}\left[X_{\mathbb{C}}\right]^{G}$ corresponds to the complex algebraic subset $Z:=V\left(\left\{P \in \mathbb{C}\left[z_{1}, \ldots, z_{m}\right] \mid P\left(p_{1}, \ldots, p_{m}\right)=0\right\}\right)$ of $\mathbb{C}^{m}$ and the inclusion $\mathbb{C}\left[X_{\mathbb{C}}\right]^{G} \subset \mathbb{C}\left[X_{\mathbb{C}}\right]$ corresponds to the polynomial map

$$
\begin{array}{clc}
X_{\mathbb{C}} \subset \mathbb{C}^{d} & \rightarrow & Z \subset \mathbb{C}^{m} \\
x & \mapsto & \left(p_{1}(x), \ldots, p_{m}(x)\right)
\end{array}
$$

The morphism $\pi$ is a finite map ([27] I.5.3 Example 1), hence surjective ([27] I.5.3 Theorem 4).

Finally, we shall consider the image $Y:=\pi(X)$ of $X$ by $\pi$. Since $\pi$ is given by real polynomials, $Y$ is a semialgebraic subset of $\mathbb{R}^{m}$ ([4] Chap. 2). Notice also that, if we denote by $W$ the Zariski closure of $Y$ in $\mathbb{R}^{m}$, then $Z=W_{\mathbb{C}}$ (see [18] Lemma 1.3). 
Definition 2.1. We call the above defined map $\pi: X \rightarrow Y$ the geometric quotient of $X$ by $G$. By abuse of terminology, we will also call $Y$ the geometric quotient of $X$ by $G$.

Remark 2.2.

- A (direct) correspondence between the real algebraic set $W$ and the real finitely generated $\mathbb{R}$-algebra $\mathcal{P}(X)^{G}$ is given by the real Nullstellensatz ([4] Theorem 4.1.4) : $\mathcal{P}(W)=$ $\mathcal{P}(X)^{G}$.

- Different sets of generators for $\mathcal{P}(X)^{G}$ provides isomorphic geometric quotients, via (real) polynomial mappings (consider the complexified algebra $\mathbb{C}[Z]=\mathcal{P}(X)^{G} \otimes_{\mathbb{R}} \mathbb{C}$ : two different sets of real invariant generators provide isomorphic algebraic sets via real polynomial mappings). Also, if $X^{\prime}$ is a real algebraic set equipped with a polynomial action of $G$ which is equivariantly polynomially isomorphic to $X$, then $\mathcal{P}\left(X^{\prime}\right)^{G} \cong \mathcal{P}(X)^{G}$ and $X$ and $X^{\prime}$ have isomorphic quotients. As a conclusion, the geometric quotient is well-defined up to polynomial isomorphism.

- If $x_{1}, x_{2} \in X$ then $\pi\left(x_{1}\right)=\pi\left(x_{2}\right)$ if and only if there exists $g \in G$ such that $x_{2}=\alpha_{g}\left(x_{1}\right)$ (see for instance [27] Chapter 1, section 2.3, Example 11).

- Let $H$ be another finite group. If we let $X^{\prime} \subset \mathbb{R}^{d^{\prime}}$ be an algebraic set equipped with a polynomial action of $H$ and $\pi^{\prime}: X^{\prime} \rightarrow Y^{\prime} \subset \mathbb{R}^{m^{\prime}}$ be the corresponding quotient map, then the quotient map corresponding to the induced action of $G \times H$ on $X \times X^{\prime}$ is

$$
\pi \times \pi^{\prime}: \begin{array}{rlc}
X \times X^{\prime} & \rightarrow & Y \times Y^{\prime} \\
\left(x, x^{\prime}\right) & \mapsto & \left(\pi(x), \pi^{\prime}\left(x^{\prime}\right)\right)
\end{array}
$$

(this follows from the isomorphism of $\mathbb{R}$-algebra $\left.\mathcal{P}\left(X \times X^{\prime}\right)^{G \times H} \cong \mathcal{P}(X)^{G} \otimes_{\mathbb{R}} \mathcal{P}\left(X^{\prime}\right)^{H}\right)$.

Example 2.3. 1. Consider the action of $G:=\mathbb{Z} / 2 \mathbb{Z}$ on $X:=\mathbb{R}^{2}$ given by the involution $\sigma:\left(x_{1}, x_{2}\right) \mapsto\left(-x_{1}, x_{2}\right)$. Then $\mathcal{P}(X)=\mathbb{R}\left[X_{1}, X_{2}\right], \mathcal{P}(X)^{G}=\mathbb{R}\left\langle X_{1}^{2}, X_{2}\right\rangle$ and $\pi:\left(x_{1}, x_{2}\right) \mapsto\left(x_{1}^{2}, x_{2}\right)$, so that the geometric quotient of $X$ by $G$ is the half-plane $\left\{\left(y_{1}, y_{2}\right) \in \mathbb{R}^{2} \mid y_{1} \geq 0\right\}$.

2. Now, consider the action of $G$ on $X$ given by $\sigma:\left(x_{1}, x_{2}\right) \mapsto\left(-x_{1},-x_{2}\right)$. We have $\mathcal{P}(X)^{G}=\mathbb{R}\left\langle X_{1}^{2}, X_{2}^{2}, X_{1} X_{2}\right\rangle$ and $\pi:\left(x_{1}, x_{2}\right) \mapsto\left(x_{1}^{2}, x_{2}^{2}, x_{1} x_{2}\right)$, so that the geometric quotient of $X$ by $G$ is the half elliptic cone $\left\{\left(y_{1}, y_{2}, z\right) \in \mathbb{R}^{3} \mid z^{2}=y_{1} y_{2}, y_{1} \geq 0, y_{2} \geq 0\right\}$.

We give some basic properties of the previous construction. The proofs are analogous to the complex case. More precisely, consider the complex geometric quotient of the complexification and use the fact that the maps are given by real polynomial functions.

First, the real geometric quotient is functorial with respect to polynomial maps :

Lemma 2.4. Let $X$ and $X^{\prime}$ be $G$-real algebraic sets and let $\pi: X \rightarrow Y$ and $\pi^{\prime}: X^{\prime} \rightarrow Y^{\prime}$ be the respective geometric quotients of $X$ and $X^{\prime}$ by $G$. If $\psi: X \rightarrow X^{\prime}$ is an equivariant polynomial map, there exists a unique polynomial map $\rho: Y \rightarrow Y^{\prime}$ such that the following diagram

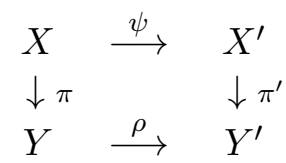


commutes.

If we have an equivariant inclusion of real algebraic sets $X \subset X^{\prime}$, the geometric quotient of $X$ can also be naturally embedded in the geometric quotient of $X^{\prime}$ :

Lemma 2.5. Keep the notation of previous lemma 2.4 and suppose that we have an equivariant inclusion $X \subset X^{\prime}$. It induces an equivariant inclusion $Y \subset Y^{\prime}$ of the corresponding geometric quotients : the geometric quotient of $X$ is (up to polynomial isomorphim) the image of $X$ under the quotient map of $X^{\prime}$.

Example 2.6. 1. Consider the action of $G:=\mathbb{Z} / 2 \mathbb{Z}$ on the unit circle $\mathbb{S}^{1}$ given by the involution $\sigma:\left(x_{1}, x_{2}\right) \mapsto\left(-x_{1}, x_{2}\right)$. We then apply the quotient map $\pi$ of example 2.3 (i) to obtain that the geometric quotient of $\mathbb{S}^{1}$ by $G$ is the semialgebraic subset $\left\{\left(y_{1}, y_{2}\right) \in \mathbb{R}^{2} \mid y_{1}+y^{2}=1, y_{1} \geq 0\right\}$.

2. Now, consider the free action of $G$ on $\mathbb{S}^{1}$ given by the involution $\sigma:\left(x_{1}, x_{2}\right) \mapsto\left(-x_{1},-x_{2}\right)$. We apply the quotient map $\pi$ of example 2.3 (ii) and the geometric quotient of $\mathbb{S}^{1}$ by $G$ is the section of the half elliptic cone by the hyperplane of equation $y_{1}+y_{2}=1$, hence an ellipse (it is then polynomially isomorphic to the unit circle).

3. Consider the free action of $G$ on the hyperbola $X:=\left\{x_{1} x_{2}=1\right\}$ of $\mathbb{R}^{2}$ given by the same involution $\sigma:\left(x_{1}, x_{2}\right) \mapsto\left(-x_{1},-x_{2}\right)$. The geometric quotient of $X$ by $G$ is the section of the half elliptic cone by the hyperplane $y_{3}=1$, hence the "half-hyperbola" $\left\{y_{1} y_{2}=1, y_{1}, y_{2} \geq 0, y_{3}=1\right\}$ (polynomially isomorphic to the half-hyperbola $\left\{x_{1} x_{2}=\right.$ $\left.1, x_{1}, x_{2} \geq 0\right\}$ of $\mathbb{R}^{2}$ ).

We will finally consider some geometric properties of the quotient : we begin by checking that the geometric quotient preserves the dimension. Again, let $X$ denote a $G$-real algebraic set and $\pi: X \rightarrow Y \subset W$ be the associated quotient map, where $W$ is the Zariski closure of $Y$.

Lemma 2.7. We have

$$
\operatorname{dim} Y=\operatorname{dim} W=\operatorname{dim} X .
$$

Proof. The left-hand side equality follows from the fact that $W$ is the Zariski closure of $Y$ ([4] Proposition 2.8.2). To establish the right-hand side equality, we use the fact that the dimension of $X$ as a real algebraic set is equal to the dimension of $X_{\mathbb{C}}$ as a complex algebraic set (see [1] II.2, in particular Proposition 2.2.1.d and Proposition 2.2.5.b). But $\operatorname{dim} X_{\mathbb{C}}=\operatorname{dim} Z$ because the geometric quotient map $\pi: X_{\mathbb{C}} \rightarrow Z$ is a finite map (see [27] I.5.3 Example 1). Since $Z=W_{\mathbb{C}}$, we obtain $\operatorname{dim} W=\operatorname{dim} X$.

We will also use the essential fact that the image under the quotient map of a nonsingular point with trivial stabilizer is nonsingular :

Proposition 2.8. Let $x$ be a nonsingular point of $X$. If $G_{x}=\{e\}$, then $\pi(x)$ is a nonsingular point of $W$.

Proof. The proof is analogous to the complex case : see for instance the proof of [27] II.2.1 Example. 
The map $\pi$ induces an isomorphism

$$
m_{\pi(x)} / m_{\pi(x)}^{2} \rightarrow m_{x} / m_{x}^{2}
$$

between the duals of the respective Zariski tangent spaces of $W$ at $\pi(x)$ and of $X$ at $x$. As a consequence :

Proposition 2.9. Suppose that $x$ is a nonsingular point of $X$ and that $G_{x}=\{e\}$. Then there exist a semialgebraic open neighborhood $U$ of $x$ in $X$ and a semialgebraic open neighborhood $U^{\prime}$ of $\pi(x)$ in $W$ such that $\pi_{\mid U}$ is a Nash (i.e. semialgebraic and analytic) diffeomorphism from $U$ to $U^{\prime}$.

Proof. The point $\pi(x)$ is a nonsingular point of $W$ and $\pi$ induces an isomorphism

$$
T_{x} X \rightarrow T_{\pi(x)} W
$$

We use Proposition 8.1.2 of [4] to conclude.

\subsection{Quotient of a semialgebraic set by a polynomial finite group action}

We can restrict the geometric quotient of a $G$-real algebraic set to any of its subsets. We are more precisely interested in the restriction to semialgebraic subsets.

So let now $X \subset \mathbb{R}^{d}$ be a semialgebraic set, and suppose that the Zariski closure $\bar{X}^{\mathcal{Z}}$ of $X$ is equipped with a polynomial action of $G$ which globally stabilizes $X$ ( $G$ still denotes a finite group).

Let $\pi: \bar{X}^{\mathcal{Z}} \rightarrow Y$ be the geometric quotient of $\bar{X}^{\mathcal{Z}}$ by $G$.

Definition 2.10. We call the restriction $\pi: X \rightarrow \pi(X)$, or simply $\pi(X)$, the geometric quotient of $X$ by $G$.

Remark 2.11. As $\pi\left(\bar{X}^{\mathcal{Z}}\right)$, the geometric quotient of $X$ by $G$ is well-defined up to polynomial isomorphism (see remark 2.2) and $\operatorname{dim} \pi(X)=\operatorname{dim} X$ (see lemma 2.7). Moreover, if the action of $G$ on $\bar{X}^{\mathcal{Z}}$ comes from a polynomial action on a real algebraic set $X^{\prime}$ containing $\bar{X}^{\mathcal{Z}}$, the geometric quotient of $X$ can be obtained as the image of $X$ under the quotient map of $X^{\prime}$ (see lemma 2.5).

Example 2.12. Consider the action of the involution $\sigma:\left(x_{1}, x_{2}\right) \mapsto\left(-x_{1}, x_{2}\right)$ on the upper half-plane $\left\{x_{2} \geq 0\right\}$ of $\mathbb{R}^{2}$. Its geometric quotient is the first quadrant $\left\{y_{1} \geq 0, y_{2} \geq 0\right\}$ of $\mathbb{R}^{2}$ (see example 2.3).

Lemma 2.13. The continuous map $\pi: X \rightarrow \pi(X)$ is proper, closed and open (with respect to the Euclidean topology). Moreover, it is homeomorphic to the topological quotient map $q: X \rightarrow X / G$.

In order to prove this lemma, we will suppose without loss of generality that $X$ is globally stabilized under an orthogonal linear action : 
Lemma 2.14. Let $V \subset \mathbb{R}^{d}$ be a $G$-real algebraic set. There exists an equivariant polynomial isomorphism $\varphi: V \rightarrow V^{\prime}$, where $V^{\prime} \subset \mathbb{R}^{D}$ is a real algebraic set equipped with a linear action of $G$ on $\mathbb{R}^{D}$ given by permutation matrices.

Proof. Indeed, denote $G:=\left\{g_{1}, \ldots, g_{N}\right\}$ where $g_{1}$ is the identity element of $G$ and consider the morphism

$$
\varphi: \begin{array}{ccc}
V & \rightarrow & V \times \cdots \times V \\
x & \mapsto & \left(x, \alpha_{g_{2}^{-1}}(x), \ldots, \alpha_{g_{N}^{-1}}(x)\right)
\end{array}
$$

Now, equip the cartesian product $V \times \cdots \times V$ with the action of $G$ given by the permutations induced by the product in $G$. The morphism $\varphi$ is then equivariant.

Furthermore, $\varphi$ induces a polynomial isomorphism between $V$ and its image $\varphi(V)$ : the direct image of $V$ by $\varphi$ is an algebraic subset of $\mathbb{R}^{d} \times \cdots \times \mathbb{R}^{d}$ given by the equations $y_{1} \in V$, $y_{i}=\alpha_{g_{i}}\left(y_{1}\right)$ (recall that each $\alpha_{i}$ is a polynomial isomorphism).

Proof of lemma 2.13. Up to polynomial isomorphism, we can then suppose that $X$ is globally stabilized by a linear orthogonal action of $G$ on $\mathbb{R}^{d}$, and $\pi: X \rightarrow \pi(X)$ is then the restriction of the corresponding quotient map $\pi=\left(p_{1}, \ldots, p_{m}\right): \mathbb{R}^{d} \rightarrow \mathbb{R}^{m}$ on $\mathbb{R}^{d}$ (lemma 2.5).

The map $\pi: \mathbb{R}^{d} \rightarrow \mathbb{R}^{m}$ is proper. Indeed, let us recall the argument of [26] : the map

$$
\phi: \begin{array}{lll}
\mathbb{R}^{d} & \rightarrow & \mathbb{R} \\
x=\left(x_{1}, \ldots, x_{d}\right) & \mapsto\langle x, x\rangle=x_{1}^{2}+\ldots+x_{d}^{2}
\end{array}
$$

is a proper polynomial map, which is invariant under right composition with the orthogonal action of $G$ on $\mathbb{R}^{d}$. Therefore, $\phi \in \mathcal{P}\left(\mathbb{R}^{d}\right)^{G}$ and there exists a polynomial $Q \in \mathbb{R}\left[Y_{1}, \ldots, Y_{m}\right]$ such that $\phi=Q\left(p_{1}, \ldots, p_{m}\right)=Q \circ \pi$. Now, let $K$ be a compact set in $\mathbb{R}^{m}$. Then $Q(K)$ is a compact of $\mathbb{R}$ and $\phi^{-1}(Q(K))$ is a compact set of $\mathbb{R}^{d}$ (because $\phi$ is proper). Finally, $\pi^{-1}(K)$ is a closed subset of $\mathbb{R}^{d}$ (because $\pi$ is continuous) included in $\phi^{-1}(Q(K))$, hence it is compact as well.

The restriction of a proper map being a proper map, the map $\pi: X \rightarrow \pi(X)$ is also proper.

Since $\mathbb{R}^{m}$ is a Hausdorff locally compact space, the proper map $\pi: \mathbb{R}^{d} \rightarrow \mathbb{R}^{m}$ is closed. Now, consider a closed subset $F \cap X$ of $X$, where $F$ is a closed subset of $\mathbb{R}^{d}$. Since $X$ is stable under the action of $G, \pi(F \cap X)=\pi(F) \cap \pi(X)$ and this is a closed subset of $\pi(X)$ since $\pi(F)$ is a closed subset of $\mathbb{R}^{m}$ ( $\pi$ is closed on $\mathbb{R}^{d}$ ). Consequently, $\pi: X \rightarrow \pi(X)$ is a closed map.

It is open as well. Indeed, take $U$ to be an open subset of $X$, then $\pi(U)=\pi\left(\bigcup_{g \in G} g \cdot U\right)$ so we can assume $U$ to be globally stable under the action of $G$. Now, $\pi(X \backslash U)$ is a closed subset of $\pi(X)$, but $\pi(X \backslash U)=\pi(X) \backslash \pi(U)$ (since $U$ is stable under the action of $G$ ) therefore $\pi(U)$ is an open subset of $\pi(X)$.

Finally, let us show that the map $\pi: X \rightarrow \pi(X)$ is homeomorphic to the topological quotient $\operatorname{map} q: X \rightarrow X / G$.

The map $\pi: X \rightarrow \pi(X)$ is a continuous surjective map such that if $x, y \in X, \pi(x)=\pi(y)$ if and only if there exists $g \in G$ such that $x=g \cdot y$. As a consequence, there exists a continuous bijective map $\bar{\pi}: X / G \rightarrow \pi(X)$ such that $\pi=\bar{\pi} \circ q$. The map $\bar{\pi}$ is a homeomorphism since $\pi$ is an open map. 


\subsection{Construction of the quotient of an arc-symmetric set by a free finite group action}

From now, we will consider $\mathcal{A S}$-sets of $\mathbb{P}^{d}(\mathbb{R})$, in the sense of [12] (Definition 3.1 and Definition 2.7). We recall below the definitions of an arc-symmetric set of $\mathbb{P}^{d}(\mathbb{R})$ and of an $\mathcal{A S}$-set of $\mathbb{P}^{d}(\mathbb{R})$ :

Definition 2.15. Let $A$ be a subset of $\mathbb{P}^{d}(\mathbb{R})$.

- $A$ is called an arc-symmetric set of $\mathbb{P}^{d}(\mathbb{R})$ if $A$ is semialgebraic and if, for every real analytic arc $\gamma:(-1,1) \rightarrow \mathbb{P}^{d}(\mathbb{R})$, the inclusion $\gamma((-1,0)) \subset A$ implies the entire inclusion $\gamma((-1,1)) \subset A$.

- $A$ is called an $\mathcal{A S}$-set $X$ of $\mathbb{P}^{d}(\mathbb{R})$ if $A$ is Boolean combination of arc-symmetric sets of $\mathbb{P}^{d}(\mathbb{R})$.

Remark 2.16. - The arc-symmetric sets of $\mathbb{P}^{d}(\mathbb{R})$ are closed (with respect to the strong topology of $\mathbb{P}^{d}(\mathbb{R})$ ) hence compact (the arc-symmetric sets of $\mathbb{P}^{d}(\mathbb{R})$ are the closed $\mathcal{A S}$ sets of $\left.\mathbb{P}^{d}(\mathbb{R})\right)$. If $A$ is any subset of $\mathbb{P}^{d}(\mathbb{R})$, the intersection of all arc-symmetric sets of $\mathbb{P}^{d}(\mathbb{R})$ containing $A$ is the smallest arc-symmetric set of $\mathbb{P}^{d}(\mathbb{R})$ containing $A$, and it is denoted by $\bar{A}^{\mathcal{A S}}$ (we call $\bar{A}^{\mathcal{A S}}$ the $\mathcal{A S}$-closure of $A$ ).

- A semialgebraic set $A \subset \mathbb{P}^{d}(\mathbb{R})$ is an $\mathcal{A S}$-set if and only if, for every real analytic arc $\gamma:(-1,1) \rightarrow \mathbb{P}^{d}(\mathbb{R})$ such that $\gamma((-1,0)) \subset A$, there exists $\epsilon>0$ such that $\gamma((0, \epsilon)) \subset A$.

- The real algebraic sets, or more generally any Zariski open subset of a real algebraic set, are $\mathcal{A S}$-sets, as well as their compact connected components.

Definition 2.17. We call a $G$ - $\mathcal{A S}$-set any $\mathcal{A S}$-set equipped with an action of $G$ via homeomorphisms with $\mathcal{A S}$-graph.

Remark 2.18. Let $X$ and $Y$ be $\mathcal{A S}$-sets and let $f: X \rightarrow Y$ be a map with $\mathcal{A S}$-graph : we will call such a map an $\mathcal{A S}$-map. If $f$ is injective, then $f(X)$ is an $\mathcal{A S}$-set (Theorem 3.4 of [12]). If $f$ is bijective, then $f^{-1}$ has $\mathcal{A S}$-graph.

Our goal in this section is to define a well-defined $\mathcal{A S}$-quotient for some particular $G$ - $\mathcal{A S}$ sets. First, if $X \subset \mathbb{P}^{d}(\mathbb{R})$ is any $G$ - $\mathcal{A S}$-set, we will embed it in such a way that the geometric quotient can be considered :

Proposition 2.19. Let $X \subset \mathbb{P}^{d}(\mathbb{R})$ be a $G$-AS-set. Then there exist a $G$-AS-set $S$ and an equivariant $\mathcal{A S}$-homeomorphism $\varphi: X \rightarrow S$ such that

1. $S$ is an $\mathcal{A S}$-set contained in an affine space $\mathbb{R}^{D} \subset \mathbb{P}^{D}(\mathbb{R})$ (we will simply say that $S$ is an $\mathcal{A S}$-set of $\mathbb{R}^{D}$ ),

2. the action of $G$ on $S$ is the restriction of an orthogonal action of $G$ on $\mathbb{R}^{D}$.

3. the Zariski closure $\bar{S}^{\mathcal{Z}}$ of $S$ in $\mathbb{R}^{D}$ is compact. 
Such an embedding will be called a $G$-orthogonal embedding of the $G$-AS-set $X$. Furthermore, a $G$-AS-set which verifies the three above properties will be called a $G$-orthogonal $\mathcal{A S}$-set.

Proof. The real algebraic variety $\mathbb{P}^{d}(\mathbb{R})$ can be biregularly embedded into a compact algebraic subset of $\mathbb{R}^{D_{0}}:=\mathbb{R}^{(d+1)^{2}}$ ([4], Theorem 3.4.4). Via this embedding, we equivariantly embed $X$ as a $G$ - $\mathcal{A S}$-set $S_{0}$ of $\mathbb{R}^{D_{0}}$, where $S_{0}$ is equipped with the action of $G$ obtained by composition with the biregular embedding and its inverse. Notice that the (projective) Zariski closure of $X$ in $\mathbb{P}^{d}(\mathbb{R})$ is embedded as the Zariski closure of $S_{0}$ in $\mathbb{R}^{D_{0}}$, which is then compact.

Now, apply the $\mathcal{A S}$-version of lemma 2.14 to embed $S_{0}$, via an equivariant $\mathcal{A S}$-homeomorphism, as a $G$ - $\mathcal{A S}$-subset $S$ of a product of $N:=|G|$ copies of $S_{0}$ on which the action of $G$ is given by permutation matrices.

Finally, notice that the Zariski closure of $S$ is globally preserved by this linear action on $\mathbb{R}^{D}:=\left(\mathbb{R}^{D_{0}}\right)^{N}$ (since so is $S$ ) and that it is included in the product of $N$ copies of $\overline{S_{0}} \mathcal{Z}$, therefore it is compact as well.

We can now consider the image of the semialgebraic set $S$ under the quotient map $\pi: \mathbb{R}^{D} \rightarrow$ $Y \subset \mathbb{R}^{m}$. Notice that this quotient depends on the considered $G$-orthogonal embedding of $X$. Nevertheless we will see in proposition 2.29 below that, if $X$ is compact and the action of $G$ on $X$ is free, then two $G$-orthogonal embeddings of $X$ induce $\mathcal{A S}$-homeomorphic quotients of $X$.

If $S$ is compact (i.e. $X$ is compact) and the action of $G$ is free, then $\pi(S)$ is also a compact arc-symmetric set : we recall the proof of this important result of [10] (Proposition 3.15).

Proposition 2.20. Suppose that $S$ is compact (that is $S$ is an arc-symmetric set of $\mathbb{R}^{D}$ ) and that, for all $x \in S, G_{x}=\{e\}$. Then $\pi(S)$ is an arc-symmetric set of $\mathbb{R}^{m}$.

Proof. $\pi(S)$ is a semialgebraic subset of $\mathbb{R}^{m}$ as the image of a semialgebraic set by the polynomial map $\pi$. It is furthermore compact since $S$ is compact and $\pi$ is continuous.

Now, considering the standard embedding $\mathbb{R}^{m} \subset \mathbb{P}^{m}(\mathbb{R})$, we are going to prove that $\pi(S)$ is an $\mathcal{A S}$-set of $\mathbb{P}^{m}(\mathbb{R})$. Consider a real analytic map $\gamma:(-1,1) \rightarrow \mathbb{P}^{m}(\mathbb{R})$ such that $\gamma((-1,0)) \subset$ $\pi(S)$. First, notice that $y:=\gamma(0) \in \pi(S)$ because $\pi(S)$ is closed. Let $x \in S \subset \mathbb{R}^{D}$ such that $y=\pi(x)$. Since $x$ is a nonsingular point of $\mathbb{R}^{D}$ and $G_{x}=\{e\}$, by proposition 2.9 , there exists a semialgebraic open neighborhood $U$ of $x$ in $\mathbb{R}^{D}$ and a semialgebraic open neighborhood $U^{\prime}$ of $y=\pi(x)$ in the Zariski closure $W$ of $\pi\left(\mathbb{R}^{D}\right)$, such that $\pi_{\mid U}$ is a Nash diffeomorphism from $U$ to $U^{\prime}$. Denote by $\eta$ the inverse map of $\pi_{\mid U}$.

Let $\epsilon^{\prime}>0$ such that $\gamma\left(\left(-\epsilon^{\prime}, 0\right]\right) \subset U^{\prime}$. Composing $\gamma_{\mid\left(-\epsilon^{\prime}, 0\right]}$ with $\eta$, we obtain an analytic map $\eta \circ \gamma:\left(-\epsilon^{\prime}, 0\right] \rightarrow U \subset \mathbb{R}^{d}$ that we can extend into an analytic map $\widetilde{\gamma}:\left(-\epsilon^{\prime}, \epsilon\right) \rightarrow U$, with $0<\epsilon<1$. Since $\gamma\left(\left(-\epsilon^{\prime}, 0\right]\right) \subset \pi(S)$, we have $\widetilde{\gamma}\left(\left(-\epsilon^{\prime}, 0\right]\right)=\eta \circ \gamma\left(\left(-\epsilon^{\prime}, 0\right]\right) \subset S$ and, since $S$ is arc-symmetric, $\widetilde{\gamma}\left(\left(-\epsilon^{\prime}, \epsilon\right)\right) \subset S$.

We finally apply $\pi$ to obtain a real analytic arc $\pi \circ \tilde{\gamma}:\left(-\epsilon^{\prime}, \epsilon\right) \rightarrow \mathbb{R}^{m} \subset \mathbb{P}^{m}(\mathbb{R})$ which coincides with the real analytic arc $\gamma:(-1,1) \rightarrow \mathbb{P}^{m}(\mathbb{R})$ on $\left(-\epsilon^{\prime}, 0\right)$. As a consequence, by analytic continuation, $\gamma\left(\left(-\epsilon^{\prime}, \epsilon\right)\right)=\pi \circ \widetilde{\gamma}\left(\left(-\epsilon^{\prime}, \epsilon\right)\right) \in \pi(S)$. As a conclusion, $\pi(S)$ is an $\mathcal{A S}$-set of $\mathbb{P}^{m}(\mathbb{R})$.

Since $\pi(S)$ is compact, it is an arc-symmetric set of $\mathbb{P}^{m}(\mathbb{R})$ and, since $\pi(S) \subset \mathbb{R}^{m}, \pi(S)$ is an arc-symmetric set of $\mathbb{R}^{m}$ (see also Remark 3.5 of [12]). 
Remark 2.21. 1. Even if $S$ is an algebraic set, the quotient $\pi(S)$ is not algebraic in general. Consider the example of Remark 3.16 of [10] : the quotient of the compact algebraic subset $\left\{y^{2}+\left(x^{2}-2\right)\left(x^{2}-1\right)\left(x^{2}+1\right)=0\right\}$ of $\mathbb{R}^{2}$ by the free involution $(x, y) \mapsto(-x, y)$ is the compact connected component $\{x \geq 0\}$ of the algebraic set $\left\{y^{2}+(x-2)(x-1)(x+1)=0\right\}$ (it is a non-algebraic arc-symmetric set).

2. If the action of $G$ on $S$ is free but $S$ is not compact, the quotient $\pi(S)$ is not an $\mathcal{A S}$-set in general. Consider the third example of 2.6 : the half-hyperbola is not an $\mathcal{A S}$-set.

We can actually establish a slightly more general result, where $S$ is not assumed to be compact, by considering the compact $\mathcal{A S}$-closure $\bar{S}^{\mathcal{A S}}$ of $S$ (notice that it is included in the Zariski closure $\bar{S}^{\mathcal{Z}} \subset \mathbb{R}^{D}$ of $S$ ) :

Corollary 2.22. Suppose that the action of $G$ on $\bar{S}^{\mathcal{A S}} \subset \mathbb{R}^{D}$ is free. Then $\pi(S)$ is an $\mathcal{A S}$-set of $\mathbb{R}^{m}$.

Proof. First, notice that the action of $G$ on $\mathbb{R}^{D}$ globally stabilizes $\bar{S}^{\mathcal{A S}}$ as well (because it stabilizes $S$ and the image of a compact $\mathcal{A S}$-set by an $\mathcal{A S}$-homeomorphism is a compact $\mathcal{A S}$ set).

Now, even if $S$ is not compact, $\bar{S}^{\mathcal{A S}}$ is. As a consequence, since for all $x \in \bar{S}^{\mathcal{A S}}, G_{x}=\{e\}$, $\pi\left(\bar{S}^{\mathcal{A S}}\right)$ is a compact arc-symmetric subset of $\mathbb{R}^{m}$ by proposition 2.20 .

We then proceed by induction on the dimension of $S$ using that $\operatorname{dim}\left(\bar{S}^{\mathcal{A S}} \backslash S\right)<\operatorname{dim}(S)$ (Proposition 3.3 of [12]) and that $\pi\left(\bar{S}^{\mathcal{A S}} \backslash S\right)=\pi\left(\bar{S}^{\mathcal{A S}}\right) \backslash \pi(S)$ i.e. $\pi(S)=\pi\left(\bar{S}^{\mathcal{A S}}\right) \backslash$ $\pi\left(\bar{S}^{\mathcal{A S}} \backslash S\right)$.

Definition 2.23. If the action of $G$ on $\bar{S}^{\mathcal{A S}}$ is free, we will say that the G-orthogonal embedding $S$ of $X$ is a free $G$-orthogonal embedding of $X$.

If $X$ possesses a free $G$-orthogonal-embedding, we will say that $X$ is a free $G$-AS-set.

\subsection{Functoriality and uniqueness of the quotient of a free $G$ - $\mathcal{A S}$-set}

In the last part of this section, we establish functoriality properties of the previous construction. In particular, this will allow to prove that the quotient of a free $G$ - $\mathcal{A S}$-set is well-defined up to $\mathcal{A S}$-homeomorphism.

We begin with an equivariant continuous $\mathcal{A S}$-map $f: X \rightarrow X^{\prime}$ between two free $G$ - $\mathcal{A S}$ sets. We then consider a free $G$-orthogonal embedding $\varphi: X \rightarrow S \subset \mathbb{R}^{D}$ of $X$ and a free $G$-orthogonal embedding $\varphi^{\prime}: X^{\prime} \rightarrow S^{\prime} \subset \mathbb{R}^{D^{\prime}}$ of $X^{\prime}$.

If $\pi: \mathbb{R}^{D} \rightarrow Y$ and $\pi^{\prime}: \mathbb{R}^{D^{\prime}} \rightarrow Y^{\prime}$ are the respective geometric quotients of $\mathbb{R}^{D}$ and $\mathbb{R}^{D^{\prime}}$ by $G$, we define a map

$$
f_{/ G}: \pi(S) \rightarrow \pi^{\prime}\left(S^{\prime}\right)
$$


between the $\mathcal{A S}$-sets $\pi(S)$ and $\pi^{\prime}\left(S^{\prime}\right)$, such that the following diagram commutes :

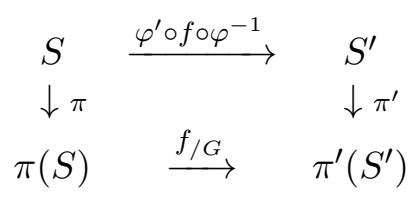

Precisely, if $y=\pi(x) \in \pi(S)$, we set $f_{/ G}(y):=\pi^{\prime}\left(\varphi^{\prime} \circ f \circ \varphi^{-1}(x)\right)$ (notice that this definition is independent of the chosen preimage of $y$ since $f$ is equivariant and because the fiber of $\pi$ at $y$ is the orbit of $x$ under the action of $G$ on $S$ ).

Proposition 2.24. The above defined map $f_{/ G}: \pi(S) \rightarrow \pi^{\prime}\left(S^{\prime}\right)$ is continuous.

Proof. Let $y=\pi(x) \in \pi(S)$. Denote by $W$ the Zariski closure of $Y$. Since $x$ is a nonsingular point of $\mathbb{R}^{d}$ and $G_{x}=\{e\}$, according to proposition 2.9 , there exist a semialgebraic open neighborhood $U$ of $x$ in $\mathbb{R}^{d}$ and a semialgebraic open neighborhood $U^{\prime}$ of $\pi(x)$ in $W$ such that $\pi_{\mid U}$ is a Nash diffeomorphism from $U$ to $U^{\prime}$. As a consequence, on the open neighborhood $U^{\prime} \cap \pi(S)$ of $y, f_{/ G}$ can be described as $\pi^{\prime} \circ f \circ \pi_{\mid U}^{-1}$, hence the result (recall that $\pi^{\prime}$ is a polynomial map and $f$ is continuous).

Remark 2.25. If the embeddings $\varphi$ and $\varphi^{\prime}$ are $\mathcal{C}^{k}$-diffeomorphisms with $k \in \mathbb{N}, k=\infty$ or $k=\omega$ and $f$ is a $\mathcal{C}^{k}$-map, one can show, using the same argument, that $f_{/ G}$ is a $\mathcal{C}^{k}$-map as well.

We now show that the graph of $f_{/ G}$ is an $\mathcal{A S}$-set, by describing it as the image of a free $(G \times G)-\mathcal{A S}$-set by a geometric quotient :

Proposition 2.26. Suppose $Y \subset \mathbb{R}^{m}$ and $Y^{\prime} \subset \mathbb{R}^{m^{\prime}}$. The graph of $f_{/ G}$ is an $\mathcal{A S}$-set of $\mathbb{R}^{m+m^{\prime}}$.

Proof. The graph $\Gamma_{f_{/ G}}$ of $f_{/ G}$ is

$$
\Gamma_{f_{/ G}}=\left\{\left(\pi(x), \pi^{\prime}\left(x^{\prime}\right)\right) \in Y \times Y^{\prime} \mid x \in S, x^{\prime} \in S^{\prime}, x^{\prime}=f(x)\right\} .
$$

Hence, it is the image of the graph $\Gamma_{f}$ of $f$ under the geometric quotient $\pi \times \pi^{\prime}: \mathbb{R}^{D} \times \mathbb{R}^{D^{\prime}} \rightarrow$ $Y \times Y^{\prime}$ of $\mathbb{R}^{D} \times \mathbb{R}^{D^{\prime}}$ under the product action of $G \times G$ (see remark 2.2).

Notice that $\Gamma_{f}$ is not globally stable under the action of $G \times G$. However, we also have

$$
\Gamma_{f_{/ G}}=\pi\left(\bigcup_{\left(g, g^{\prime}\right) \in G \times G} \alpha_{g} \times \alpha_{g^{\prime}}\left(\Gamma_{f}\right)\right),
$$

the union $\bigcup_{\left(g, g^{\prime}\right) \in G \times G} \alpha_{g} \times \alpha_{g^{\prime}}\left(\Gamma_{f}\right)$ being a $(G \times G)-\mathcal{A S}$-set of $\mathbb{R}^{D} \times \mathbb{R}^{D^{\prime}}$. Since the arcsymmetric closure of this $\mathcal{A S}$-set is included in $\bar{S}^{\mathcal{A S}} \times{\overline{S^{\prime}}}^{\mathcal{A S}}$, we conclude by corollary 2.22 .

Remark 2.27. If $f$ is a proper map, so is $f_{/ G}$. Indeed, let $K$ be a compact subset of $\pi^{\prime}\left(X^{\prime}\right)$, then

$$
f_{/ G}^{-1}(K)=\pi\left(\left(\varphi^{\prime} \circ f \circ \varphi^{-1}\right)^{-1}\left(\pi^{\prime-1}(K)\right)\right)
$$

is a compact subset of $\pi(X)$ since $\pi^{\prime}$ is proper (lemma 2.13), $f$ is proper, $\varphi$ and $\varphi^{\prime}$ are homeomorphisms and $\pi$ is continuous. 
Finally, the operation which associates to $f$ the continuous $\mathcal{A S}$-map $f_{/ G}$ is functorial :

Proposition 2.28. Let $X^{\prime \prime}$ be a free $G$-AS-set and $\varphi^{\prime \prime}: X^{\prime \prime} \rightarrow S^{\prime \prime}$ be a free $G$-orthogonal embedding of $X^{\prime \prime}$, and let $h: X^{\prime} \rightarrow X^{\prime \prime}$ be an equivariant continuous $\mathcal{A S}$-map.

Then the equivariant continuous composition $h \circ f: S \rightarrow S^{\prime \prime}$ has $\mathcal{A S}$-graph and

$$
(h \circ f)_{/ G}=h_{/ G} \circ f_{/ G} .
$$

Furthermore, if $i d_{X}$ denotes the identity map on $X$, then $\left(i d_{X}\right)_{/ G}=i d_{\pi(S)}$.

Proof. Let $\pi^{\prime \prime}: \mathbb{R}^{d^{\prime \prime}} \rightarrow Y^{\prime \prime}$ be the geometric quotient of $\mathbb{R}^{d^{\prime \prime}}$ by $G$. Then, for any $\pi(x) \in \pi(S)$,

$$
h_{/ G} \circ f_{/ G}(\pi(x))=h_{/ G}\left(\pi^{\prime}\left(\left(\varphi^{\prime} \circ f \circ \varphi^{-1}\right)(x)\right)\right)=\pi^{\prime \prime}\left(\left(\varphi^{\prime \prime} \circ h \circ \varphi^{\prime-1}\right) \circ\left(\varphi^{\prime} \circ f \circ \varphi^{-1}\right)(x)\right) .
$$

Proposition 2.29. Let $X$ be a free $G$-AS-set. Let $\varphi: X \rightarrow S$ and $\varphi^{\prime}: X \rightarrow S^{\prime}$ be two free $G$-orthogonal embeddings of $X$. Then the equivariant $\mathcal{A S}$-homeomorphism $\varphi^{\prime} \circ \varphi^{-1}$ induces an $\mathcal{A S}$-homeomorphism between the geometric quotients of $S$ and $S^{\prime}$, which are $\mathcal{A S}$-sets.

As a consequence, we will call the geometric quotient of any free $G$-orthogonal embedding of $X$ "the" $\mathcal{A S}$-quotient of the free $G$-AS-set $X$, well-defined up to $\mathcal{A S}$-homeomorphism.

Remark 2.30. If two free $G$ - $\mathcal{A S}$-sets are equivariantly $\mathcal{A S}$-homeomorphic, then they have $\mathcal{A S}$ homeomorphic $\mathcal{A S}$-quotients.

If $f$ is an equivariant continuous $\mathcal{A S}$-map between two free $G$ - $\mathcal{A S}$-sets, then the induced continuous $\mathcal{A S}$-map $f / G$ between the corresponding $\mathcal{A S}$-quotients is well-defined up to $\mathcal{A S}$ homeomorphism.

Remark 2.31. For the definition of the $\mathcal{A S}$-quotient of a free $G$ - $\mathcal{A S}$-set $X$, we could have considered more general embeddings, namely any equivariant $\mathcal{A S}$-embedding of $X$ as a $\mathcal{A S}$-set $V$ globally stabilized under a polynomial action of $G$ on an affine space, such that the action is free on the $\mathcal{A S}$-closure of $V$. The geometric quotient of such an embedding also realizes the $\mathcal{A S}$-quotient of $X$.

\section{Equivariant homologies and cohomologies}

Keep $G$ to be a finite group. From now on, we want to consider and study invariants of $G$ - $\mathcal{A S}$ sets. First, we choose the "equivariant" homologies and cohomologies with which we are going to work.

\subsection{Equivariant singular homology and cohomology}

Let $X$ be a topological space on which $G$ acts via homeomorphisms : we will call such a space a $G$-space. We consider the equivariant singular homology

$$
H_{*}^{G}(X):=H_{*}\left(X \times_{G} E_{G}\right)
$$


and equivariant singular cohomology

$$
H_{G}^{*}(X):=H^{*}\left(X \times_{G} E_{G}\right)
$$

of $X$ with coefficients in $\mathbb{F}_{2}$, defined using the Borel construction ([5]) :

- $H_{*}(\cdot):=H_{*}\left(\cdot, \mathbb{F}_{2}\right)$ and $H^{*}(\cdot):=H^{*}\left(\cdot, \mathbb{F}_{2}\right)$ stand for the singular homology and cohomology with coefficients in $\mathbb{F}_{2}$,

- $E_{G}$ is the total space of a universal principal $G$-bundle $E_{G} \rightarrow B_{G}$,

- $X \times_{G} E G$ denotes the topological quotient of the product space $X \times E G$ by the diagonal action of $G$.

Remark 3.1. 1. The equivariant singular homology and cohomology are independent of the choice of a universal $G$-bundle.

2. Let $E$ be a contractible topological space equipped with a free action of $G$. Since $G$ is a finite group, the action of $G$ on $E$ is proper and the quotient map $E \rightarrow E / G$ is a universal principal $G$-bundle.

3. Since $E_{G}$ is a contractible space, $X \times E_{G}$ is equivariantly homotopic to $X$ and $X \times{ }_{G} E_{G}=$ $\left(X \times E_{G}\right) / G$ is called the homotopy quotient of $X$ by $G$.

4. The equivariant map $X \rightarrow\{p t\}$ induces a fibration $X \times{ }_{G} E_{G} \rightarrow\{p t\} \times E_{G} / G=B_{G}$ with fiber $X$. In particular, we have Leray-Serre spectral sequences

$$
E_{p, q}^{2}=H_{p}\left(B_{G}, H_{q}(X)\right) \Rightarrow H_{p+q}^{G}(X)
$$

and

$$
E_{2}^{p, q}=H^{p}\left(B_{G}, H^{q}(X)\right) \Rightarrow H_{G}^{p+q}(X)
$$

(see for instance [14] section 11.4).

5. If $G=\{e\}, H_{*}^{G}(X)=H_{*}(X)$. If $X$ is contractible (e.g. if $X$ is a point), $H_{*}^{G}(X)=$ $H_{*}\left(B_{G}\right)=H_{*}\left(G, \mathbb{F}_{2}\right)$, since $G$ is finite, where $H_{*}\left(G, \mathbb{F}_{2}\right)$ is the homology of the group $G$ with coefficients in $\mathbb{F}_{2}$ (see for instance [6]). If the action of $G$ on $X$ is trivial, $H_{*}^{G}(X)=H_{*}(X) \otimes_{\mathbb{F}_{2}} H_{*}\left(G, \mathbb{F}_{2}\right)$ (in this case, $X \times_{G} E_{G}=X \times B_{G}$ and we use the Künneth isomorphism in homology). Finally, if the action of $G$ on $X$ is free, we have $H_{*}^{G}(X)=H_{*}(X / G)$ (in this case, the equivariant map $E_{G} \rightarrow\{p t\}$ induces a fibration $X \times_{G} E_{G} \rightarrow X / G$ with fiber $E_{G}$, which is contractible). We have similar statements for the cohomological counterparts.

6. If $X$ is a $G-C W$-complex, i.e. if $X$ is a $C W$-complex and the action of $G$ permutes its cells, the equivariant singular homology and cohomology coincide respectively with the equivariant homology and cohomology defined in [6] Chap. VII, sect. 7. Indeed, consider a contractible $G-C W$-complex $E$ such that $G$ freely permutes its cells (see for instance the construction of [11] Example 1B.7). We have an equivariant chain isomorphism

$$
C_{*}^{\text {cell }}(E) \otimes_{\mathbb{F}_{2}} C_{*}^{\text {cell }}(X) \rightarrow C_{*}^{\text {cell }}(E \times X),
$$


where $C_{*}^{\text {cell }}(\cdot)$ denotes the cellular chain complex with coefficients in $\mathbb{F}_{2}$, which induces a chain isomorphism

$$
C_{*}^{\text {cell }}(E) \otimes_{G} C_{*}^{\text {cell }}(X) \rightarrow C_{*}^{\text {cell }}(E \times X)_{G} \rightarrow C_{*}^{\text {cell }}((E \times X) / G)
$$

(see [6] Chap. III sect. 0 and Chap. II Proposition 2.4), which itself induces an isomorphism in equivariant homology (the cellular complex of the homotopy quotient $(E \times X) / G$ computes its homology, and $C_{*}^{\text {cell }}(E)$ is a free resolution of $\mathbb{Z}$ over $\mathbb{Z}[G]$ since $E$ is a free $G-C W$-complex : see [6] Chap. I Proposition 4.1).

As for the cohomological counterpart, apply the duality functor $\operatorname{Hom}_{\mathbb{F}_{2}}\left(\cdot, \mathbb{F}_{2}\right)$ to the above chain isomorphism and use the tensor-hom adjunction to obtain natural isomorphisms

$$
\operatorname{Hom}_{\mathbb{F}_{2}}\left(C_{p}^{\text {cell }}(E) \otimes_{G} C_{q}^{\text {cell }}(X), \mathbb{F}_{2}\right) \cong \operatorname{Hom}_{G}\left(C_{p}^{\text {cell }}(E), \operatorname{Hom}_{\mathbb{F}_{2}}\left(C_{q}^{\text {cell }}(X), \mathbb{F}_{2}\right)\right) .
$$

\subsection{A real algebraic model for the total space $E_{G}$}

In order to study the equivariant geometry of $G$ - $\mathcal{A S}$-sets via these equivariant homology and cohomology, we choose for $E_{G}$ a suitable "real algebraic" model : for $n \in \mathbb{N} \backslash\{0\}$ and $k \in$ $\mathbb{N} \cup\{\infty\}$, consider the Stiefel manifold $V_{n}\left(\mathbb{R}^{k}\right)$, which is the set of the orthonormal $n$-frames of $\mathbb{R}^{k}$, that is $n$-tuples of orthonormal vectors of $\mathbb{R}^{k}$ (recall that $\mathbb{R}^{\infty}$ denotes the space of sequences $\left(x_{n}\right)_{n \in \mathbb{N} \backslash\{0\}}$ such that $x_{n} \neq 0$ for finitely many $i$ 's).

Remark that, if $k$ is finite, $V_{n}\left(\mathbb{R}^{k}\right)$ is a compact space, as a closed subspace of the product of $n$ copies of the unit sphere in $\mathbb{R}^{k}$. Besides, the natural inclusions $V_{n}\left(\mathbb{R}^{k}\right) \hookrightarrow V_{n}\left(\mathbb{R}^{k+1}\right) \hookrightarrow V_{n}\left(\mathbb{R}^{\infty}\right)$ fit into the equality

$$
V_{n}\left(\mathbb{R}^{\infty}\right)=\underset{k \in \mathbb{N}}{\lim _{\longrightarrow}} V_{n}\left(\mathbb{R}^{k}\right)
$$

(it is an infinite increasing union in $V_{n}\left(\mathbb{R}^{\infty}\right)$ ).

Furthermore, $V_{n}\left(\mathbb{R}^{\infty}\right)$ is a contractible space (see for instance Example 4.53 of [11]) and one can equip $V_{n}\left(\mathbb{R}^{\infty}\right)$, as well as any $V_{n}\left(\mathbb{R}^{k}\right)$, with a free action of $G$ :

Lemma 3.2. Let $n \in \mathbb{N} \backslash\{0\}$ and $k \in \mathbb{N} \cup\{\infty\}$, and let $H$ be any subgroup of the orthogonal group $O_{n}(\mathbb{R})$. There is a free action of $H$ on $V_{n}\left(\mathbb{R}^{k}\right)$.

Proof. Identify $V_{n}\left(\mathbb{R}^{k}\right)$ with the set of matrices $A \in M_{k, n}(\mathbb{R})$ such that ${ }^{t} A A=I_{n}$ (if $k=\infty, A$ has finitely many nonzero coefficients) and, if $M \in H$ and $A \in V_{n}\left(\mathbb{R}^{k}\right)$, set $M \cdot A:=A M^{-1} \in$ $V_{n}\left(\mathbb{R}^{k}\right)$ (we have $\left.{ }^{t}\left(A M^{-1}\right) A M^{-1}={ }^{t}\left(M^{-1}\right)^{t} A A M^{-1}=I_{n}\right)$.

This action of $H$ on $V_{n}\left(\mathbb{R}^{k}\right)$ is free since, if $M \in H, A \in V_{n}\left(\mathbb{R}^{k}\right), A M=A$ implies $M=I_{n}$.

Remark 3.3. We can describe the action of $H$ on $V_{n}\left(\mathbb{R}^{k}\right)$ as a restriction of a linear action of $H$ on $\left(\mathbb{R}^{n}\right)^{k}$. Indeed, if $A=\left(v_{1}, \ldots, v_{n}\right) \in V_{n}\left(\mathbb{R}^{k}\right)$, write $v_{i}=\left(a_{1, i}, \ldots, a_{k, i}\right)$ and associate to $\left(v_{1}, \ldots, v_{n}\right)$ the single vector

$$
v:=\left(a_{1,1}, \ldots, a_{1, n}, \ldots, a_{k, 1}, \ldots, a_{k, n}\right) .
$$

Now, let $M$ be an element of $H$ and $M_{0}$ be the block diagonal matrix with $k$ copies of $M$ as diagonal blocks. Then, via this correspondence, the action of $M$ on $A$ corresponds to the multiplication of the vector $v \in\left(\mathbb{R}^{n}\right)^{k}$ by the matrix $M_{0}\left(\right.$ recall that $\left.M^{-1}={ }^{t} M\right)$. 
Denote $N:=|G|$. By giving indices to the elements of the finite group $G$, we can define an injective group homomorphism from $G$ into the subgroup of permutation matrices of the orthogonal group $O_{N}(\mathbb{R})$. For the rest of the paper, fix such an embedding.

Then the quotient map $V_{N}\left(\mathbb{R}^{\infty}\right) \rightarrow V_{N}\left(\mathbb{R}^{\infty}\right) / G$ is a universal principal $G$-bundle. Moreover :

Lemma 3.4. For any $n \in \mathbb{N} \backslash\{0\}$ and $k \in \mathbb{N}$, the Stiefel manifold $V_{n}\left(\mathbb{R}^{k}\right)$ is a compact nonsingular algebraic subset of $\mathbb{R}^{n k}$.

Proof. The set $V_{n}\left(\mathbb{R}^{k}\right) \subset\left(\mathbb{R}^{n}\right)^{k}$ is described by the real algebraic equations

$$
P_{i}:=a_{1, i}^{2}+\ldots+a_{k, i}^{2}-1=0, \quad 1 \leq i \leq n
$$

and

$$
Q_{i, j}:=a_{1, i} a_{1, j}+\ldots+a_{k, i} a_{k, j}=0, \quad 1 \leq i<j \leq n,
$$

and the columns of the matrix of the partial derivatives of the polynomials $P_{i}$ 's and $Q_{i, j}$ 's are linearly independent at any point $\left(a_{i, j}\right)_{i, j}$ of $V_{n}\left(\mathbb{R}^{k}\right)$ (otherwise we would have a nontrivial linear relation between the orthonormal vectors $\left(a_{1, j}, \ldots, a_{n, j}\right), 1 \leq j \leq n$, which is impossible).

The set $V_{n}\left(\mathbb{R}^{k}\right)$ is therefore a nonsingular real algebraic set of dimension $n k-\frac{n(n+1)}{2}$.

Now let $X$ be $G-\mathcal{A S}$-set. We are going to realize the equivariant singular homology

$$
H_{*}^{G}(X)=H_{*}\left(X \times_{G} V_{N}\left(\mathbb{R}^{\infty}\right)\right)
$$

of $X$ as an inductive limit of the singular homologies of the $(\mathcal{A S}$ - $)$ quotients of $X \times V_{N}\left(\mathbb{R}^{k}\right)$ by $G$.

First, fix a $G$-orthogonal embedding of $X$ (proposition 2.19), so that we can assume $X$ to be a $G$-orthogonal $\mathcal{A S}$-subset of $\mathbb{R}^{d}$ (the topological quotient map $X \times V_{N}\left(\mathbb{R}^{\infty}\right) \rightarrow$ $\left(X \times V_{N}\left(\mathbb{R}^{\infty}\right)\right) / G$ and the corresponding topological quotient map for the embedding are the same up to homeomorphism).

Now, let $k \in \mathbb{N}$. The Stiefel manifold $V_{N}\left(\mathbb{R}^{k}\right)$ is acted by an orthogonal action of $G$ on $\mathbb{R}^{N k}$ (remark 3.3), and $X \times V_{N}\left(\mathbb{R}^{k}\right)$ is an $\mathcal{A S}$-subset of $\mathbb{R}^{d+N k}$ globallly stabilized under the induced diagonal orthogonal action of $G$ on $\mathbb{R}^{d+N k}$.

Denote $N_{k}:=d+N k$ and fix $p_{1}, \ldots, p_{m}$ to be generators of the corresponding invariant algebra $\mathbb{R}\left[X_{1}, \ldots, X_{N_{k}}\right]^{G}$. The action of $G$ on $X \times V_{N}\left(\mathbb{R}^{k+1}\right) \subset \mathbb{R}^{N_{k+1}}$ is the diagonal (orthogonal) action of $G$ on $\mathbb{R}^{N_{k}} \times \mathbb{R}^{N}$ (remark 3.3). We can then suppose the generators of the invariant algebra $\mathbb{R}\left[X_{1}, \ldots, X_{N_{k}}, X_{N_{k}+1}, \ldots, X_{N_{k}+N}\right]^{G}$ to be the polynomials $p_{1}, \ldots, p_{m}$ together with polynomials $q_{1}, \ldots, q_{m^{\prime}} \in \mathbb{R}\left[X_{1}, \ldots, X_{N_{k}}, X_{N_{k}+1}, \ldots, X_{N_{k}+N}\right]^{G}$ such that $q_{j}\left(X_{1}, \ldots, X_{N_{k}}, 0, \ldots, 0\right)=0$ for $j \in\left\{1, \ldots, m^{\prime}\right\}$.

If we denote by $i$ the natural (equivariant) embedding of $\mathbb{R}^{N_{k}}$ in $\mathbb{R}^{N_{k}+N}$, it induces, by functoriality of the real geometric quotient (lemma 2.4), the following commutative diagram

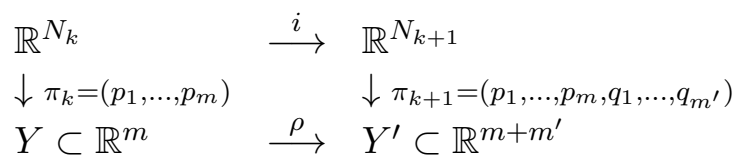


of geometric quotients, where $\rho: \begin{array}{ccc}Y & \rightarrow & Y^{\prime} \\ \left(y_{1}, \ldots, y_{m}\right) & \mapsto & \left(y_{1}, \ldots, y_{m}, 0, \ldots, 0\right)\end{array}$. Notice that, if we denote by $y_{1}, \ldots, y_{m}, z_{1}, \ldots, z_{m^{\prime}}$ the coordinates in $\mathbb{R}^{m+m^{\prime}}$, then $\rho(Y)=Y^{\prime} \cap\left\{z_{1}=\ldots=z_{m^{\prime}}=\right.$ $0\}$.

Finally, we restrict to the inclusion $i: X \times V_{N}\left(\mathbb{R}^{k}\right) \hookrightarrow X \times V_{N}\left(\mathbb{R}^{k+1}\right)$ to obtain the commutative diagram

$$
\begin{array}{ccc}
X \times V_{N}\left(\mathbb{R}^{k}\right) & \stackrel{i}{\longrightarrow} & X \times V_{N}\left(\mathbb{R}^{k+1}\right) \\
\downarrow \pi_{k} & & \\
\pi_{k}\left(X \times V_{N}\left(\mathbb{R}^{k}\right)\right) \subset \mathbb{R}^{m} & \stackrel{\rho}{\longrightarrow} & \pi_{k+1}\left(X \times V_{N}\left(\mathbb{R}^{k+1}\right)\right) \subset \mathbb{R}^{m+m^{\prime}}
\end{array}
$$

In particular, $\rho\left(\pi_{k}\left(X \times V_{N}\left(\mathbb{R}^{k}\right)\right)\right)=\pi_{k+1}\left(X \times V_{N}\left(\mathbb{R}^{k+1}\right)\right) \cap\left\{z_{1}=\ldots=z_{m^{\prime}}=0\right\}$. Remark that the $\mathcal{A S}$-closure of $X \times V_{N}\left(\mathbb{R}^{k}\right)$ is $\bar{X}^{\mathcal{A S}} \times V_{N}\left(\mathbb{R}^{k}\right)$ (use the induction on dimension together with Proposition 3.3 of [12]), on which the diagonal action of $G$ is free. As a consequence, the geometric quotient $\pi_{k}\left(X \times V_{N}\left(\mathbb{R}^{k}\right)\right)$ of $X \times V_{N}\left(\mathbb{R}^{k}\right)$ by $G$ is an $\mathcal{A S}$-set of $\mathbb{R}^{m}$ (corollary 2.22) : it is the $\mathcal{A S}$-quotient of $X \times V_{N}\left(\mathbb{R}^{k}\right)$ (proposition 2.29).

The maps $\pi_{k}: X \times V_{N}\left(\mathbb{R}^{k}\right) \rightarrow \pi_{k}\left(X \times V_{N}\left(\mathbb{R}^{k}\right)\right)$ form an inductive system which induces a map

$$
\Pi: X \times V_{N}\left(\mathbb{R}^{\infty}\right)=\varliminf_{k \in \mathbb{N}} X \times V_{N}\left(\mathbb{R}^{k}\right) \rightarrow \underset{k \in \mathbb{N}}{\lim _{k}} \pi_{k}\left(X \times V_{N}\left(\mathbb{R}^{k}\right)\right) .
$$

Since each map $\pi_{k}$ is continuous, closed, open and verifies, for $x, y \in X \times V_{N}\left(\mathbb{R}^{k}\right), \pi_{k}(x)=$ $\pi_{k}(y)$ if and only if there exists $g \in G$ such that $x=g \cdot y$, the map $\Pi$ is continuous, closed, open and verifies for $x, y \in X \times V_{N}\left(\mathbb{R}^{\infty}\right), \Pi(x)=\Pi(y)$ if and only if there exists $g \in G$ such that $x=g \cdot y$ as well.

As a consequence, the surjective map

$$
\Pi: X \times V_{N}\left(\mathbb{R}^{\infty}\right) \rightarrow \underset{k \in \mathbb{N}}{\lim _{\vec{x}}} \pi_{k}\left(X \times V_{N}\left(\mathbb{R}^{k}\right)\right)
$$

is, up to homeomorphism, the topological quotient map $X \times V_{N}\left(\mathbb{R}^{\infty}\right) \rightarrow\left(X \times V_{N}\left(\mathbb{R}^{\infty}\right)\right) / G$. Therefore,

$$
H_{*}^{G}(X)=H_{*}\left(\lim _{k \in \mathbb{N}} \pi_{k}\left(X \times V_{N}\left(\mathbb{R}^{k}\right)\right)\right) .
$$

We then use Proposition 3.33 of [11] to establish the following statement :

Proposition 3.5. Denote $X_{(k)}:=\pi_{k}\left(X \times V_{N}\left(\mathbb{R}^{k}\right)\right)$. The inductive system of inclusions $X_{(k)} \rightarrow X_{(k+1)}$ induces an isomorphism

$$
\underset{k \in \mathbb{N}}{\lim _{k}} H_{*}\left(X_{(k)}\right) \rightarrow H_{*}^{G}(X) .
$$

Proof. The inductive limit $\lim _{k \in \mathbb{N}} X_{(k)}$ can be considered as an increasing union in $\mathbb{R}^{\infty}$. Let $K$ be a compact of $\lim _{k \in \mathbb{N}} X_{(k)}$. Then $K$ is a compact of $\mathbb{R}^{\infty}$. 
Now, if $l \in \mathbb{N}$, consider the $C W$-complex structure on $\mathbb{R}^{l}$ whose 0 -dimensional cells are the points of $\mathbb{Z}^{l}$ and the higher dimensional open cells are the open hypercubes of edge-length one and vertices in $\mathbb{Z}^{l}$. These $C W$-complex structures on $\mathbb{R}^{l}, l \in \mathbb{N}$, are compatible with the natural inclusions $\mathbb{R}^{l} \rightarrow \mathbb{R}^{l+1}$, so that $\mathbb{R}^{l}$ is a subcomplex of $\mathbb{R}^{l+1}$. Moreover, via these inclusions, they induce a $C W$-complex structure on $\mathbb{R}^{\infty}$, such that each $\mathbb{R}^{l}$ is a subcomplex of $\mathbb{R}^{\infty}$.

By, for instance, Proposition A.1 of [11], the compact set $K$ is then included in a finite subcomplex of $\mathbb{R}^{\infty}$. Therefore, it is included in some $\mathbb{R}^{l}$ for $l \in \mathbb{N}$ and there exists $k_{0} \in \mathbb{N}$ such that $K \subset \mathbb{R}^{m_{k_{0}}}$, where $m_{k_{0}}$ is the number of coordinate polynomial functions of $\pi_{k_{0}}$. Finally,

$$
K \subset \underset{k \in \mathbb{N}}{\lim _{\overrightarrow{\mathbb{N}}}} X_{(k)} \cap \mathbb{R}^{m_{k_{0}}}=X_{\left(k_{0}\right)}
$$

and we conclude by Proposition 3.33 of [11].

Dually, we obtain :

Corollary 3.6. The inductive system of inclusions $X_{(k)} \rightarrow X_{(k+1)}$ induces a projective system in singular cohomology $H^{*}\left(X_{(k+1)}\right) \rightarrow H^{*}\left(X_{(k)}\right)$ and an isomorphism

$$
H_{G}^{*}(X) \rightarrow \lim _{k \in \mathbb{N}} H^{*}\left(X_{(k)}\right) .
$$

Proof. We have

$$
\begin{aligned}
H_{G}^{*}(X) & \cong \operatorname{Hom}_{\mathbb{F}_{2}}\left(H_{*}^{G}(X), \mathbb{F}_{2}\right) \\
& \cong \operatorname{Hom}_{\mathbb{F}_{2}}\left(\underset{k \in \mathbb{N}}{\lim _{*}} H_{*}\left(X_{(k)}\right), \mathbb{F}_{2}\right) \\
& =\lim _{k \in \mathbb{N}} \operatorname{Hom}_{\mathbb{F}_{2}}\left(H_{*}\left(X_{(k)}\right), \mathbb{F}_{2}\right) \\
& \cong \lim _{k \in \mathbb{N}} H^{*}\left(X_{(k)}\right)
\end{aligned}
$$

(see also Proposition 3F.5 of [11] and its proof).

\subsection{Equivariant homology with closed supports and equivariant cohomology with compact supports}

We now define another equivariant homology for the $G$ - $\mathcal{A S}$-set $X$ using the semialgebraic chain complexes with closed supports and coefficients in $\mathbb{F}_{2}$ (see [16], Appendix) of the $\mathcal{A S}$-sets $X_{(k)}$, $k \in \mathbb{N}$. Precisely, the natural inclusions $X_{(k)} \rightarrow X_{(k+1)}$ induce an inductive system of injective chain morphisms

$$
C_{*}\left(X_{(k)}\right) \rightarrow C_{*}\left(X_{(k+1)}\right)
$$

and we denote by $C_{*}(X ; G)$ its inductive limit.

As for the cohomological counterpart, the inclusions $X_{(k)} \rightarrow X_{(k+1)}$ induce a projective system of surjective cochain morphisms

$$
C^{*}\left(X_{(k+1)}\right) \rightarrow C^{*}\left(X_{(k)}\right),
$$


where $C^{*}$ denote the dual cochain complex of $C_{*}$ (see [13], section 2.3), and we denote by $C^{*}(X ; G)$ its projective limit.

Notice that the complexes $C_{*}(X ; G)$ and $C^{*}(X ; G)$ do not depend, up to (co)chain complex isomorphism, on the chosen $G$-orthogonal embedding of $X$ because the semialgebraic chain complex with closed supports is functorial with respect to proper continuous semialgebraic maps ([16], Appendix) and the $\mathcal{A S}$-quotient of a free $G-\mathcal{A S}$-set is unique up to $\mathcal{A S}$-homeomorphism (proposition 2.29).

Definition 3.7. We define by

$$
H_{*}(X ; G):=H_{*}\left(C_{*}(X ; G)\right)
$$

and

$$
H^{*}(X ; G):=H^{*}\left(C^{*}(X ; G)\right)
$$

the respective equivariant homology with closed supports and equivariant cohomology with compact supports of $X$ and coefficients in $\mathbb{F}_{2}$.

Remark 3.8. - Because homology commutes with inductive limits (see for instance [28] Chap. 4, Sec. 1, Theorem 7) and since the semialgebraic chain complex with closed supports computes Borel-Moore homology, we have

$$
H_{*}(X ; G)=\underset{k \in \mathbb{N}}{\lim _{*}} H_{*}^{B M}\left(X_{(k)}\right) .
$$

- We also have

$$
H^{*}(X ; G)=\operatorname{Hom}_{\mathbb{F}_{2}}\left(H_{*}(X ; G), \mathbb{F}_{2}\right)=\lim _{k \in \mathbb{N}} H_{c}^{*}\left(X_{(k)}\right):
$$

the left Hom functor of an inductive limit is the projective limit of the Hom functors (see for instance the proof of Proposition 3F.5 of [11]), and the dual semialgebraic cochain complex computes the cohomology with compact supports (see [13], section 2.3).

- The equivariant homology with closed supports $H_{*}(X ; G)$ of $X$ is different from the equivariant homology considered in [20] and [23]. When $X$ is compact, the equivariant cohomology with compact supports $H^{*}(X ; G)$ of $X$ coincides with the equivariant cohomology considered in [23] : see remark 3.10 below.

Lemma 3.9. If the $G$-AS-set $X$ is compact, then $H_{*}(X ; G)=H_{*}^{G}(X)$ and $H^{*}(X ; G)=$ $H_{G}^{*}(X)$.

Proof. If $X$ is compact, so is each quotient set $X_{(k)}$ (as the image of a compact set by a continuous map) and we have isomorphisms $H_{*}^{B M}\left(X_{(k)}\right) \rightarrow H_{*}\left(X_{(k)}\right)$, such that the diagrams

$$
\begin{array}{ccc}
H_{*}^{B M}\left(X_{(k)}\right) & \longrightarrow & H_{*}^{B M}\left(X_{(k+1)}\right) \\
\downarrow \cong & & \downarrow \cong \\
H_{*}\left(X_{(k)}\right) & \longrightarrow & H_{*}\left(X_{(k+1)}\right)
\end{array}
$$


are commutative. As a consequence, the inductive systems $H_{*}^{B M}\left(X_{(k)}\right), k \in \mathbb{N}$ and $H_{*}\left(X_{(k)}\right)$, $k \in \mathbb{N}$ are isomorphic and the induced direct limits are isomorphic.

Composing with the duality functor $\operatorname{Hom}_{\mathbb{F}_{2}}\left(\cdot, \mathbb{F}_{2}\right)$ provides the isomorphism $H^{*}(X ; G) \cong$ $H_{G}^{*}(X)$.

Remark 3.10. By [19], $X$ has a (unique) semialgebraic $G$ - $C W$-structure so that, if $X$ is compact, $H_{*}(X ; G)$ coincides with the equivariant cohomology of [6] Chap. VII, sect. 7 (see remark 3.1 (6) above), that is the homology $H_{*}\left(G, C_{*}^{c e l l}(X)\right)$ of the group $G$ with coefficients in the chain complex $C_{*}^{\text {cell }}(X)$.

On the other hand, since $G$ is a finite group, $X$ admits a (unique) $G$-equivariant semialgebraic triangulation (which induces its semialgebraic $G$-CW-structure) and we can use it to relate the chain complexes $C_{*}(X)$ and $C_{*}^{c e l l}(X)$ via an equivariant quasi-isomorphism.

Consequently, if $X$ is compact, $H_{*}(X ; G)=H_{*}\left(G, C_{*}(X)\right)$ ([6] Chap. VII, Proposition 5.2) and, by dualization, $H^{*}(X ; G)=H^{*}\left(G, C^{*}(X)\right)$ (this was the definition of the equivariant cohomology considered in [23], Definition 3.3).

Example 3.11. We compute the equivariant homology of the real 2-dimensional unit sphere $X:=\mathbb{S}^{2}$ in $\mathbb{R}^{3}$ equipped with two different kind of actions of $G:=\mathbb{Z} / 2 \mathbb{Z}$, using the spectral sequence

$$
E_{p, q}^{2}=H_{p}\left(G, H_{q}(X)\right) \Rightarrow H_{p+q}(X ; G)
$$

induced by the double complex $F_{*} \otimes_{G} C_{*}(X)$, if $F_{*}$ is a projective resolution of $\mathbb{F}_{2}$ over $\mathbb{F}_{2}[G]$ (see [6] Chap. VII, see also [20] section 3).

1. Consider the action of $G$ on $X$ given by the central symmetry $\sigma:\left(x_{1}, x_{2}, x_{3}\right) \mapsto\left(-x_{1},-x_{2},-x_{3}\right)$. The projective resolution of $\mathbb{F}_{2}$ over $\mathbb{F}_{2}[G]$ we will consider is

$$
\mathbb{F}_{2} \leftarrow \mathbb{F}_{2}[G] \stackrel{1+\sigma}{\longleftarrow} \mathbb{F}_{2}[G] \stackrel{1+\sigma}{\longleftarrow} \mathbb{F}_{2}[G] \leftarrow \cdots
$$

so that the above spectral sequence is induced by the double complex

$$
C_{*}(X) \stackrel{i d_{*}+\sigma_{*}}{\longleftarrow} C_{*}(X) \stackrel{i d_{*}+\sigma_{*}}{\longleftarrow} C_{*}(X) \leftarrow \cdots
$$

We have $E^{3}=E^{2}$ and in order to compute $E^{4}$, we have to compute the image of a point $p$ by the differential $d^{3}$.

Apply $i d_{*}+\sigma_{*}$ to $\{p\}$ to obtain the union of two opposite points : they are the boundary of a half-equator. If we apply $i d_{*}+\sigma_{*}$ to this half-equator, we obtain an entire equator which is the boundary of an hemisphere. Finally, the sum of this hemisphere with its image by $\sigma_{*}$ is the entire sphere, so that the page $E^{4}$ of the spectral sequence is

$$
\begin{array}{ccccc}
0 & 0 & 0 & 0 & \cdots \\
0 & 0 & 0 & 0 & \cdots \\
\mathbb{F}_{2}[p] & \mathbb{F}_{2}[p] & \mathbb{F}_{2}[p] & 0 & \cdots
\end{array}
$$

As a consequence, 


$$
H_{k}(X ; G)= \begin{cases}\mathbb{F}_{2} & \text { if } k=0,1,2, \\ 0 & \text { otherwise. }\end{cases}
$$

Notice that, since the action on $X$ is free, we could have used the equality $H_{*}(X ; G)=$ $H_{*}(X / G)=H_{*}\left(\mathbb{P}_{2}(\mathbb{R})\right)$ (remark $\left.3.1(5)\right)$.

2. If we consider any non-free action of $G$ on $X$, we can represent the 0-homology of $X$ by a fixed point. The image of this fixed point by $i d_{*}+\sigma_{*}$ is 0 , so $E^{2}=E^{\infty}$ and

$$
H_{k}(X ; G)= \begin{cases}\mathbb{F}_{2} & \text { if } k=0,1, \\ \mathbb{F}_{2} \oplus \mathbb{F}_{2} & \text { if } k \geq 2 .\end{cases}
$$

3. More generally, the equivariant homology of the real $d$-dimensional unit sphere $\mathbb{S}^{d}$ of $\mathbb{R}^{d+1}$ by a continuous $\mathcal{A S}$-action of $G$ is

$$
H_{k}\left(\mathbb{S}^{d} ; G\right)= \begin{cases}\mathbb{F}_{2} & \text { if } 0 \leq k \leq d, \\ 0 & \text { otherwise }\end{cases}
$$

if the action is free, and

$$
H_{k}(X ; G)= \begin{cases}\mathbb{F}_{2} & \text { if } 0 \leq k \leq d-1, \\ \mathbb{F}_{2} \oplus \mathbb{F}_{2} & \text { if } k \geq d,\end{cases}
$$

if there is at least one fixed point.

\section{The $G$-Nash constructible filtrations}

In this section, for $G$ a finite group, we construct invariants for $G$ - $\mathcal{A} \mathcal{S}$-sets with respect to equivariant $\mathcal{A S}$-homeomorphisms. Precisely, for any $G$ - $\mathcal{A S}$-set $X$, we begin by constructing a filtration $\mathcal{N}_{\bullet}$ on the chain complex $C_{*}(X ; G)$ using the Nash constructible filtration of [16]. This filtered complex $\mathcal{N}_{\bullet} C_{*}(X ; G)$ is functorial with respect to equivariant $\mathcal{A S}$-homeomorphisms, as well as the induced spectral sequence $E(X ; G)$. From this spectral sequence $E(X ; G)$, we extract invariants with values in $\mathbb{Z}$ which are additive with respect to equivariant inclusions of $G$ - $\mathcal{A S}$ sets, and coincide with the dimensions of the equivariant homology on compact nonsingular $G$ - $\mathcal{A S}$-sets : we call them the $G$-virtual Betti numbers. They are different from the equivariant virtual Betti numbers of [10]. We also show that the generating function of the $G$-virtual Betti numbers, that we call the $G$-virtual Poincaré series, is a rational function whose denominator only depends on the group cohomology of $G$.

\subsection{The homological $G$-Nash constructible filtration}

To any $\mathcal{A S}$-set $T$, we can associate its semialgebraic chain complex $C_{*}(T)$, which can be equipped with the (bounded and increasing) Nash constructible filtration $\mathcal{N}_{\bullet} C_{*}(T)$ (see [16] section 3) :

$$
0=\mathcal{N}_{-q-1} C_{q}(T) \subset \mathcal{N}_{-q} C_{q}(T) \subset \cdots \subset \mathcal{N}_{-1} C_{q}(T) \subset \mathcal{N}_{0} C_{q}(T)=C_{q}(T) .
$$


This filtration on chain level induces a filtration on the Borel-Moore homology with $\mathbb{F}_{2^{-}}$ coefficients of $T$.

The Nash constructible filtration $\mathcal{N} C_{*}$ is a functor with respect to proper continuous $\mathcal{A S}$ maps. It is additive on closed inclusions so that we can recover from the induced spectral sequence the virtual Betti numbers of $\mathcal{A S}$-sets ([8], see also [15]). If $T$ is a Zariski open subset of a real algebraic set, the filtered complex $\mathcal{N}_{\bullet} C_{*}(T)$ induces the weight spectral sequence of $T$ and its weight filtration on Borel-Moore homology (see [16] subsection 1C).

The Nash constructible filtration can also be dualized to induce a cohomological Nash filtration on the cochain complex $C^{*}(T)$ (see [13] section 4) : we denote it by $\mathcal{N}^{\bullet} C^{*}(T)$. The functor $\mathcal{N} C^{*}$ is contravariant and have the cohomological counterparts of the properties of $\mathcal{N} C_{*}$.

In this paragraph, we are going to define an equivariant analog of the Nash constructible filtration on the chain complex $C_{*}(X ; G)$, for any $G$ - $\mathcal{A S}$-set $X$.

So let $X$ be a $G$ - $\mathcal{A S}$-set. For each $k \in \mathbb{N}$, we consider the Nash constructible filtration

$$
0=\mathcal{N}_{-q-1} C_{q}\left(X_{(k)}\right) \subset \mathcal{N}_{-q} C_{q}\left(X_{(k)}\right) \subset \cdots \subset \mathcal{N}_{-1} C_{q}\left(X_{(k)}\right) \subset \mathcal{N}_{0} C_{q}\left(X_{(k)}\right)=C_{q}\left(X_{(k)}\right) .
$$

The closed inclusions $X_{(k)} \hookrightarrow X_{(k+1)}, k \in \mathbb{N}$ (see subsection 3.2 above), induce an inductive system of injections of filtered chain complexes

$$
\mathcal{N}_{\bullet} C_{*}\left(X_{(k)}\right) \rightarrow \mathcal{N}_{\bullet} C_{*}\left(X_{(k+1)}\right)
$$

(see Theorem 3.6 of $[16]$ ). We also denote by $\mathcal{N}_{\bullet}$ the induced direct limit filtration on the complex $C_{*}(X ; G)$. Notice that

- for each $p$ and $q, \mathcal{N}_{p} C_{q}(X ; G)=\lim _{k \in \mathbb{N}} \mathcal{N}_{p} C_{q}\left(X_{(k)}\right)$,

- for each $q$, we have

$$
0=\mathcal{N}_{-q-1} C_{q}(X ; G) \subset \mathcal{N}_{-q} C_{q}(X ; G) \subset \cdots \subset \mathcal{N}_{-1} C_{q}(X ; G) \subset \mathcal{N}_{0} C_{q}(X ; G)=C_{q}(X ; G),
$$

- $\mathcal{N}_{\bullet} C_{*}(X ; G)$ is a bounded filtration in the sense of [14] Theorem 2.6.

Definition 4.1. We call the filtered complex $\mathcal{N}_{\bullet} C_{*}(X ; G)$ the $G$-Nash constructible filtration of $X$.

Remark 4.2. The filtered complex $\mathcal{N}_{\bullet} C_{*}(X ; G)$ is independent of the $G$-orthogonal embedding of $X$ considered to construct the inductive system of $\mathcal{A S}$-quotients $X_{(k)}, k \in \mathbb{N}$. Indeed, an equivariant $\mathcal{A S}$-homeomorphism between two $G$-orthogonal embeddings of $X$ will induce, by functoriality of the constructions, an $\mathcal{A S}$-homeomorphism between the associated inductive systems of $\mathcal{A S}$-quotients, and the Nash constructible filtration is invariant under $\mathcal{A S}$-homeomorphism.

We are now going to show that the operation which associates to any $G$ - $\mathcal{A S}$-set its $G$-Nash constructible filtration is an additive and acyclic functor. These properties are induced by the functoriality, the additivity and the acyclicity of the Nash constructible filtration (Theorem 3.6 of $[16])$. 
Theorem 4.3. The map which associates to a $G$-AS-set $X$ its $G$-Nash constructible filtration $\mathcal{N}_{\bullet} C_{*}(X ; G)$ is a functor with respect to equivariant continuous proper $\mathcal{A S}$-maps.

Proof. Let $X$ and $Y$ be two $G$ - $\mathcal{A S}$-sets and let $f: X \rightarrow Y$ be an equivariant continuous proper $\mathcal{A S}$-map between $X$ and $Y$. Fix a $G$-orthogonal embedding of $X$ and a $G$-orthogonal embedding of $Y$ and denote again by $f: X \rightarrow Y$ the induced equivariant continuous proper $\mathcal{A S}$-map between the two $G$-orthogonal embeddings.

Let $k \in \mathbb{N}$. We consider the cartesian product map

$$
f \times i d: X \times V_{N}\left(\mathbb{R}^{k}\right) \rightarrow Y \times V_{N}\left(\mathbb{R}^{k}\right)
$$

of $f$ with the identity of $V_{N}\left(\mathbb{R}^{k}\right) . f \times i d$ is again an equivariant continuous proper map, and has $\mathcal{A S}$-graph as well (the graph of $f \times i d$ is isomorphic to the cartesian product of the graph of $f$, which is $\mathcal{A S}$, and the graph of the identity of $V_{N}\left(\mathbb{R}^{k}\right)$, which is algebraic).

Now, since the sets $X \times V_{N}\left(\mathbb{R}^{k}\right)$ and $Y \times V_{N}\left(\mathbb{R}^{k}\right)$ are free $G$ - $\mathcal{A S}$-sets, the map $f \times i d$ induces a map $f_{(k)}:=(f \times i d)_{/ G}: X_{(k)} \rightarrow Y_{(k)}$ (see subsection 2.4) which is continuous (proposition 2.24), proper (remark 2.27) and has $\mathcal{A S}$-graph (proposition 2.26). As a consequence, by functoriality of the Nash constructible filtration, it induces a filtered chain map

$$
f_{(k)_{*}}: \mathcal{N}_{\bullet} C_{*}\left(X_{(k)}\right) \rightarrow \mathcal{N}_{\bullet} C_{*}\left(Y_{(k)}\right) .
$$

Furthermore, the operation which associates to $f$ the map $f_{(k)_{*}}$ is functorial (use lemma 2.28).

By functoriality of the constructions, the maps $f_{(k)_{*}}, k \in \mathbb{N}$, are compatible with the injections $\mathcal{N}_{\bullet} C_{*}\left(X_{(k)}\right) \rightarrow \mathcal{N}_{\bullet} C_{*}\left(X_{(k+1)}\right)$ and $\mathcal{N}_{\bullet} C_{*}\left(Y_{(k)}\right) \rightarrow \mathcal{N}_{\bullet} C_{*}\left(Y_{(k+1)}\right), k \in \mathbb{N}:$ precisely, we have commutative diagrams

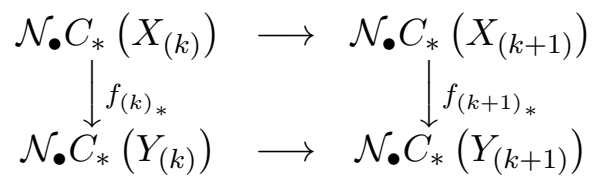

which fit into a direct limit map $f_{*}^{G}: \mathcal{N}_{\bullet} C_{*}(X ; G) \rightarrow \mathcal{N}_{\bullet} C_{*}(Y ; G)$. Of course, the operation which to $f$ associates $f_{*}^{G}$ is functorial as well, and $f_{*}^{G}$, up to a filtered chain isomorphism, does not depend on the chosen $G$-orthogonal embeddings for $X$ and $Y$.

The additivity property of the Nash constructible filtration (Theorem 3.6 of [16]) induces the additivity property of the $G$-Nash constructible filtration with respect to equivariant closed inclusions of $G-\mathcal{A S}$-sets :

Theorem 4.4. Any equivariant closed inclusion of $G$-AS-sets $Y \subset X$ induces a short exact sequence of filtered complexes

$$
0 \rightarrow \mathcal{N}_{\bullet} C_{*}(Y ; G) \rightarrow \mathcal{N}_{\bullet} C_{*}(X ; G) \rightarrow \mathcal{N}_{\bullet} C_{*}(X \backslash Y ; G) \rightarrow 0 .
$$

Proof. We fix a $G$-orthogonal embedding of $X$, which induces a $G$-orthogonal embedding of $Y$, but keep the notations $X$ and $Y$ (again, by functoriality of the construction, the result does not depend on the chosen $G$-orthogonal embedding). Let $k \in \mathbb{N}$. The equivariant closed inclusion 
$Y \subset X$ induces an equivariant closed inclusion $Y \times V_{N}\left(\mathbb{R}^{k}\right) \subset X \times V_{N}\left(\mathbb{R}^{k}\right)$. We then apply the geometric quotient map $\pi_{k}$ to obtain the closed inclusion of $\mathcal{A S}$-sets $Y_{(k)} \subset X_{(k)}$ (recall lemma 2.5 , and recall that $\pi_{k}$ is a closed map by lemma 2.13 ).

We then use the additivity of the Nash constructible filtration (Theorem 3.6 (2) of [16]) to induce the short exact sequence of filtered complexes

$$
0 \rightarrow \mathcal{N}_{\bullet} C_{*}\left(Y_{(k)}\right) \rightarrow \mathcal{N}_{\bullet} C_{*}\left(X_{(k)}\right) \rightarrow \mathcal{N}_{\bullet} C_{*}\left((X \backslash Y)_{(k)}\right) \rightarrow 0
$$

(we have $\left.X_{(k)} \backslash Y_{(k)}=(X \backslash Y)_{(k)}\right)$. We conclude by taking the direct limit : the direct limit is an exact functor on modules.

Remark 4.5. By a diagram chasing, we also have short exact sequences

$$
0 \rightarrow \frac{\mathcal{N}_{p} C_{*}(Y ; G)}{\mathcal{N}_{p-1} C_{*}(Y ; G)} \rightarrow \frac{\mathcal{N}_{p} C_{*}(X ; G)}{\mathcal{N}_{p-1} C_{*}(X ; G)} \rightarrow \frac{\mathcal{N}_{p} C_{*}(X \backslash Y ; G)}{\mathcal{N}_{p-1} C_{*}(X \backslash Y ; G)} \rightarrow 0,
$$

$p \leq 0$.

The additivity of the $G$-Nash constructible filtration implies its acyclicity :

Corollary 4.6. Let

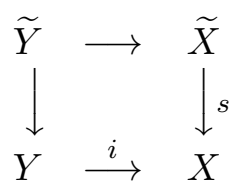

be an acyclic square of $G$-AS-sets, i.e. a commutative diagram of $G$-AS-sets and equivariant proper continuous $\mathcal{A S}$-maps such that $i$ is an equivariant closed inclusion, $\widetilde{Y}=s^{-1}(Y)$ and the restriction $s: \widetilde{X} \backslash \widetilde{Y} \rightarrow X \backslash Y$ is an equivariant homeomorphism. It induces a short exact sequence of filtered complexes

$$
0 \rightarrow \mathcal{N}_{\bullet} C_{*}(\tilde{Y} ; G) \rightarrow \mathcal{N}_{\bullet} C_{*}(Y ; G) \oplus \mathcal{N}_{\bullet} C_{*}(\tilde{X} ; G) \rightarrow \mathcal{N}_{\bullet} C_{*}(X ; G) \rightarrow 0 .
$$

Proof. The above acyclic square induces, by additivity of the $G$-Nash constructible filtration, the following commutative diagram of short exact sequences

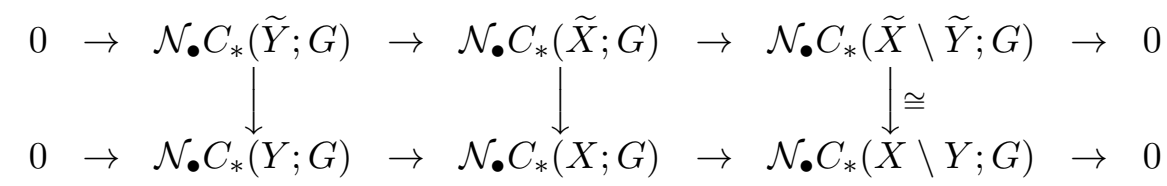

Now, the short exact sequence of the statement follows from a diagram chasing.

Remark 4.7. By a diagram chasing argument, we have short exact sequences

$$
0 \rightarrow \frac{\mathcal{N}_{p} C_{*}(\tilde{Y} ; G)}{\mathcal{N}_{p-1} C_{*}(\widetilde{Y} ; G)} \rightarrow \frac{\mathcal{N}_{p} C_{*}(Y ; G)}{\mathcal{N}_{p-1} C_{*}(Y ; G)} \oplus \frac{\mathcal{N}_{p} C_{*}(\tilde{X} ; G)}{\mathcal{N}_{p-1} C_{*}(\widetilde{X} ; G)} \rightarrow \frac{\mathcal{N}_{p} C_{*}(X ; G)}{\mathcal{N}_{p-1} C_{*}(X ; G)} \rightarrow 0
$$




\subsection{The induced $G$-weight spectral sequence}

For $X$ a $G$ - $\mathcal{A S}$-set, the filtered complex $\mathcal{N}_{\bullet} C_{*}(X ; G)$ induces a spectral sequence that we denote by $E_{*, *}^{*}(X ; G)$. Since the $G$-Nash filtration is bounded, the induced spectral sequence $E_{*, *}^{*}(X ; G)$ converges to $H_{*}\left(C_{*}(X ; G)\right)=H_{*}(X ; G)$.

Definition 4.8. We call the spectral sequence $E_{*, *}^{*}(X ; G)$ the $G$-weight spectral sequence of $X$ and we call the induced filtration

$$
0=\mathcal{N}_{-q-1} H_{q}(X ; G) \subset \mathcal{N}_{-q} H_{q}(X ; G) \subset \cdots \subset \mathcal{N}_{-1} H_{q}(X ; G) \subset \mathcal{N}_{0} H_{q}(X ; G)=H_{q}(X ; G)
$$

on the equivariant homology of $X$ with closed supports, the $G$-weight filtration of $X$.

Remark 4.9. The $G$-weight spectral sequence and filtration are different from the ones obtained in $[20]$.

The $G$-weight spectral sequence $E_{*, *}^{*}(X ; G)$ is the direct limit spectral sequence of the inductive system of spectral sequences $E_{*, *}^{*}\left(X_{(k)}\right), k \in \mathbb{N}$, where $E_{*, *}^{*}(\cdot)$ is the weight spectral sequence induced by the Nash constructible filtration. Indeed, the direct limit of the inductive system of spectral sequences induced by an inductive system of filtered complexes is the spectral sequence induced by the direct limit of the filtered complexes (use the definition of the direct limit and the exactness of the direct limit functor on modules).

In particular, for all, $r, p, q \in \mathbb{Z}$,

$$
E_{p, q}^{r}(X ; G)=\underset{k \in \mathbb{N}}{\lim _{k, q}} E_{p}^{r}\left(X_{(k)}\right) .
$$

As in [16] subsection $1 \mathrm{C}$, we reindex the spectral sequences $E_{p, q}^{r}$ into spectral sequences $\widetilde{E}_{p^{\prime}, q^{\prime}}^{r^{\prime}}$, well-defined from $r^{\prime}=1$, by setting $r^{\prime}=r+1, p^{\prime}=2 p+q, q^{\prime}=-p$. Since, for each $k \in \mathbb{N}$, the non-zero terms of $\widetilde{E}_{p, q}^{r}\left(X_{(k)}\right)$ lie in the closed triangle with vertices $(0,0),\left(0, d_{k}\right)$ and $\left(d_{k}, 0\right)$, where $d_{k}$ is the dimension of $X_{(k)}$, the spectral sequence $\widetilde{E}_{p, q}^{r}(X ; G)$ is a first quadrant spectral sequence.

We will show in theorem 4.11 below that it is right-bounded as well. First, let us mention how the additivity and acyclicity properties of the $G$-Nash constructible filtration translates on the induced $G$-weight spectral sequence :

Lemma 4.10. 1. Let $Y \subset X$ be an equivariant closed inclusion of $G$-AS-sets. For any $q \in \mathbb{N}$, it induces a long exact sequence

$$
\cdots \rightarrow \widetilde{E}_{p, q}^{2}(Y ; G) \rightarrow \widetilde{E}_{p, q}^{2}(X ; G) \rightarrow \widetilde{E}_{p, q}^{2}(X \backslash Y ; G) \rightarrow \widetilde{E}_{p-1, q}^{2}(Y ; G) \rightarrow \cdots
$$

on the $q^{\text {th }}$ line of the second page of the (reindexed) G-weight spectral sequence.

2. Consider an acyclic square (1) of $G$-AS-sets. For any $q \in \mathbb{N}$, it induces a long exact sequence

$$
\cdots \rightarrow \widetilde{E}_{p, q}^{2}(\widetilde{Y} ; G) \rightarrow \widetilde{E}_{p, q}^{2}(Y ; G) \oplus \widetilde{E}_{p, q}^{2}(\widetilde{X} ; G) \rightarrow \widetilde{E}_{p, q}^{2}(X ; G) \rightarrow \widetilde{E}_{p-1, q}^{2}(\widetilde{Y} ; G) \rightarrow \cdots
$$

on the $q^{\text {th }}$ line of the second page of the $G$-weight spectral sequence. 
Proof. These long exact sequences are induced by the short exact sequences of additivity and acyclicity of the $G$-Nash constructible filtration just as in [16] section 1C.

Theorem 4.11. Let $d$ be the dimension of $X$. If $r \geq 2$ and $\widetilde{E}_{p, q}^{r}(X ; G) \neq 0$ then $0 \leq p \leq \operatorname{dim} X$.

Proof. We will show the theorem for the $G$-orthogonal $\mathcal{A S}$-sets. Since the $G$-Nash constructible filtration of $X$ is constructed from any $G$-orthogonal embedding of $X$ (and since the dimension of a semialgebraic set is invariant under bijection with semialgebraic graph : see Theorem 2.8.8 of [4]), this will prove the result in all generality.

So assume $X$ to be a $G$-orthogonal $\mathcal{A S}$-set. We will use the induction on the dimension of $X$ (see also [10], proof of Proposition 3.10 for instance).

First, suppose that $X$ is a compact and nonsingular $\mathcal{A S}$-set, that is $X$ does not intersect the set of singular points of its Zariski closure. Then, if $k \in \mathbb{N}$, the $\mathcal{A S}$-set $X_{(k)}=\pi_{k}\left(X \times V_{N}\left(\mathbb{R}^{k}\right)\right)$ is also compact ( $\pi_{k}$ is a continuous map) and nonsingular (proposition 2.8) : recall that $V_{N}\left(\mathbb{R}^{k}\right)$ is a compact nonsingular real algebraic set (lemma 3.4). As a consequence, $X_{(k)}$ is a compact Nash submanifold of an affine space (see for instance Proposition 3.3.11 of [4]) and its weight spectral sequence, for $r \geq 2$, is then concentrated in column $p=0$ (Theorem 3.7 of [16]), i.e. $E_{p, q}^{r}\left(X_{(k)}\right)=E_{p, q}^{2}\left(X_{(k)}\right)=0$ if $p \neq 0$. Hence the same property for the direct limit : $E_{p, q}^{r}(X ; G)=E_{p, q}^{2}(X ; G)=0$ if $p \neq 0$.

This case apply in particular when $X$ is zero-dimensional, that is when $X$ is a finite union of points.

Now, suppose that $X$ is non-compact and nonsingular. There exists a compact and nonsingular $G$-orthogonal $\mathcal{A S}$-set $\bar{X}$ such that $X$ can be equivariantly biregularly embedded in $\bar{X}$ and $\operatorname{dim}(\bar{X} \backslash X)<\operatorname{dim} X$. Indeed, consider the compact Zariski closure $\bar{X}^{\mathcal{Z}}$ of $X$ (recall that $X$ is a $G$-orthogonal $\mathcal{A S}$-set), and consider an equivariant resolution of singularities $s$ of $\bar{X}^{\mathcal{Z}}$ (which exists by [3]), the action of $G$ on $s^{-1}\left(\bar{X}^{\mathcal{Z}}\right)$ being given by biregular isomorphisms. Since $X$ is away from the singularities of $\bar{X}^{\mathcal{Z}}, s^{-1}(X)$ is equivariantly biregularly isomorphic to $X$. Finally, consider an equivariant biregular embedding of $\varphi: s^{-1}\left(\bar{X}^{\mathcal{Z}}\right) \rightarrow \widetilde{X}$ into an affine space equipped with an orthogonal action of $G$ (consider the biregular versions of proposition 2.19 and lemma 2.14). If we denote $X^{\prime}:=\varphi\left(s^{-1}(X)\right)$, then ${\overline{X^{\prime}}}^{\mathcal{A S}}$ is a compact nonsingular $G$-orthogonal $\mathcal{A S}$-set $\left(\widetilde{X}\right.$ is compact and nonsingular) and $\operatorname{dim}\left({\overline{X^{\prime}}}^{\mathcal{A S}} \backslash X^{\prime}\right)<\operatorname{dim} X^{\prime}$ (Proposition 3.3 of $[12])$.

We then denote $Y:=\bar{X} \backslash X$. The $\mathcal{A S}$-closure of $Y$ is a $G$-orthogonal $\mathcal{A S}$-set as well and $\bar{Y}^{\mathcal{A S}} \cap X \subset X$ is an equivariant closed inclusion. On the other hand, $X \backslash\left(\bar{Y}^{\mathcal{A S}} \cap X\right)=$ $\bar{X} \backslash \bar{Y}^{\mathcal{A S}} \subset \bar{X}$ is an equivariant open inclusion. Consider the long exact sequence of additivity of lemma 4.10 associated to the equivariant open inclusion $X \backslash\left(\bar{Y}^{\mathcal{A S}} \cap X\right) \subset \bar{X}$ :

$\cdots \rightarrow \widetilde{E}_{p, q}^{2}\left(\bar{Y}^{\mathcal{A S}} ; G\right) \rightarrow \widetilde{E}_{p, q}^{2}(\bar{X} ; G) \rightarrow \widetilde{E}_{p, q}^{2}\left(X \backslash\left(\bar{Y}^{\mathcal{A S}} \cap X\right) ; G\right) \rightarrow \widetilde{E}_{p-1, q}^{2}\left(\bar{Y}^{\mathcal{A S}} ; G\right) \rightarrow \cdots$ 
If $p \neq 0, \widetilde{E}_{p, q}^{2}(\bar{X} ; G)=0$ by the previous case and, by the induction hypothesis, $\widetilde{E}_{p, q}^{2}\left(\bar{Y}^{\mathcal{A S}} ; G\right)=$ 0 if $p \geq d$ (recall that $\left.\operatorname{dim} \bar{Y}^{\mathcal{A S}}=\operatorname{dim} Y<\operatorname{dim} X\right)$. Consequently, if $p>d$,

$$
\widetilde{E}_{p, q}^{2}\left(X \backslash\left(\bar{Y}^{\mathcal{A S}} \cap X\right) ; G\right)=0 .
$$

Finally, consider the long exact sequence of additivity associated to the equivariant closed inclusion $\bar{Y}^{\mathcal{A S}} \cap X \subset X$ :

$\cdots \rightarrow \widetilde{E}_{p, q}^{2}\left(\bar{Y}^{\mathcal{A S}} \cap X ; G\right) \rightarrow \widetilde{E}_{p, q}^{2}(X ; G) \rightarrow \widetilde{E}_{p, q}^{2}\left(X \backslash\left(\bar{Y}^{\mathcal{A S}} \cap X\right) ; G\right) \rightarrow \widetilde{E}_{p-1, q}^{2}\left(\bar{Y}^{\mathcal{A S}} \cap X ; G\right) \rightarrow \cdots$

We use again the induction hypothesis $\left(\operatorname{dim} \bar{Y}^{\mathcal{A S}} \cap X<\operatorname{dim} X\right)$ to deduce that $\widetilde{E}_{p, q}^{2}(X ; G)=0$ if $p>d$.

We conclude the proof with the general case : if $X$ is singular, the singular points of $\bar{X}^{\mathcal{Z}}$ included in $X$ form a closed $\mathcal{A S}$-subset of $X$ which is globally stabilized under the biregular action of $G$ and of dimension stricly smaller than $\operatorname{dim} X$ (see for instance Proposition 3.3.14 of [4]). We can then use the previous cases, along with the induction hypothesis and the long exact sequence of additivity of lemma 4.10 , to conclude.

As a consequence, the long exact sequences of additivity and acyclicity of lemma 4.10 are actually finite long exact sequences. This allows us to define the following invariants :

\subsection{The $G$-virtual Betti numbers and the $G$-virtual Poincaré series}

Theorem 4.12. Let $X$ be a $G$-AS-set and let $q \in \mathbb{N}$. We denote

$$
\beta_{q}(X ; G):=\sum_{p \in \mathbb{N}}(-1)^{p} \operatorname{dim}_{\mathbb{F}_{2}} \widetilde{E}_{p, q}^{2}(X ; G)
$$

the $q^{\text {th }} G$-virtual Betti number of $X$.

The $q^{\text {th }} G$-virtual Betti number $\beta_{q}(\cdot ; G)$ has values in $\mathbb{Z}$ and is

1. an invariant of $G$ - $\mathcal{A S}$-sets with respect to equivariant $\mathcal{A S}$-homeomorphisms,

2. additive with respect to equivariant closed inclusions of $G$-AS-sets, i.e., if $Y \subset X$ is an equivariant closed inclusion, $\beta_{q}(X ; G)=\beta_{q}(Y ; G)+\beta_{q}(X \backslash Y ; G)$,

3. coincides with the dimension (over $\mathbb{F}_{2}$ ) of the $q^{\text {th }}$ equivariant homology group on (compact $G$-AS-sets equivariantly $\mathcal{A S}$-homeomorphic to) compact nonsingular $G$-orthogonal $\mathcal{A S}$ sets.

Moreover, the $q^{\text {th }} G$-virtual Betti number is unique with these properties. 
Proof. First, notice that $\beta_{q}(X ; G)$ is well-defined since the sum over $p$ is finite by theorem 4.11 .

The $q^{\text {th }} G$-virtual Betti number is invariant with respect to equivariant $\mathcal{A S}$-homeomorphisms because so are the $G$-Nash constructible filtration and the induced $G$-weight spectral sequence. It is additive because of the long exact sequence of additivity of lemma 4.10, which is finite for a given equivariant closed inclusion by theorem 4.11 .

If $X$ is a compact nonsingular $G$-orthogonal $\mathcal{A S}$-set, the $G$-weight spectral sequence of $X$ converges at $\widetilde{E}^{2}(X ; G)$ and is concentrated in the column $p=0$ (see the proof of theorem 4.11). Since the $G$-weight spectral sequence of $X$ converges to the equivariant homology of $X$ with closed supports, we have

$$
\beta_{q}(X ; G)=\operatorname{dim}_{\mathbb{F}_{2}} \widetilde{E}_{0, q}^{2}=\operatorname{dim}_{\mathbb{F}_{2}} H_{q}(X ; G)=\operatorname{dim}_{\mathbb{F}_{2}} H_{q}^{G}(X)
$$

( $X$ is compact : see lemma 3.9 ).

We finally show the uniqueness of the $q^{\text {th }} G$-virtual Betti number. Consider a map $B_{q}(\cdot ; G)$ with values in $\mathbb{Z}$ which verifies the same above properties 1$), 2)$ and 3$)$ as $\beta_{q}(\cdot ; G)$. We prove that $B_{q}(X ; G)=\beta_{q}(X ; G)$ for any $G$ - $\mathcal{A S}$-set $X$ following the steps of the proof of theorem 4.11 : we prove the result on $G$-orthogonal $\mathcal{A S}$-sets, using induction on the dimension, which implies the equality in all generality, since any $G$ - $\mathcal{A S}$-set can be embedded as a $G$-orthogonal $\mathcal{A S}$-set (and because $B_{q}(\cdot ; G)$ and $\beta_{q}(\cdot ; G)$ are invariant under equivariant $\mathcal{A S}$-homeomorphisms).

Just as in the proof of theorem 4.11, suppose first that $X$ is compact and nonsingular. Then

$$
B_{q}(X ; G)=\operatorname{dim}_{\mathbb{F}_{2}} H_{q}^{G}(X)=\beta_{q}(X ; G) .
$$

Secondly, suppose that $X$ is nonsingular and non-compact and consider an equivariant nonsingular compactification $\bar{X}$ of $X$ such that $\operatorname{dim}(\bar{X} \backslash X)<\operatorname{dim} X$. Denote $Y:=\bar{X} \backslash X$. Then $\bar{Y}^{\mathcal{A S}} \cap X \subset X$ is an equivariant closed inclusion, so

$$
B_{q}(X ; G)=B_{q}\left(\bar{Y}^{\mathcal{A S}} \cap X ; G\right)+B_{q}\left(X \backslash \bar{Y}^{\mathcal{A S}} ; G\right),
$$

and $X \backslash \bar{Y}^{\mathcal{A S}}=\bar{X} \backslash \bar{Y}^{\mathcal{A S}} \subset \bar{X}$ is an equivariant open inclusion, so that

$$
B_{q}\left(X \backslash \bar{Y}^{\mathcal{A S}} ; G\right)=B_{q}(\bar{X} ; G)-B_{q}\left(\bar{Y}^{\mathcal{A S}} ; G\right) .
$$

As a consequence, $B_{q}(X ; G)=\beta_{q}(X ; G)$ thanks to the induction hypothesis and the previous case $(\bar{X}$ is compact nonsingular).

The final step consists in considering the closed subset of singular points of $X$ and to use again the additivity of $B_{q}(\cdot ; G)$ and $\beta_{q}(\cdot ; G)$, the induction hypothesis and the previous cases.

Remark 4.13. If $X$ is a nonsingular $G$ - $\mathcal{A S}$-set and $\varphi: X \rightarrow S$ is a $G$-orthogonal embedding of $X, S$ may not be a nonsingular $\mathcal{A S}$-set.

However, if the action of $G$ on $X \subset \mathbb{P}^{d}(\mathbb{R})$ is given by biregular isomorphisms on a Zariski open subset $U$ of a (projective) algebraic set of $\mathbb{P}^{d}(\mathbb{R})$ containing $X$, the $G$-orthogonal embedding of $U$ constructed as in the proof of proposition 2.19 is a biregular isomorphism, so that the image of $X$ by this embedding is also nonsingular in this case. 
Actually, we can relax the "closed inclusion" hypothesis in the additivity property of the $G$-virtual Betti numbers :

Corollary 4.14. If $Y \subset X$ is any equivariant inclusion of $G$-AS-sets, then

$$
\beta_{q}(X ; G)=\beta_{q}(Y ; G)+\beta_{q}(X \backslash Y ; G)
$$

for all $q \in \mathbb{N}$.

Proof. Let $q \in \mathbb{N}$. Again, we begin by showing the result for $G$-orthogonal $\mathcal{A S}$-sets.

We show that

$$
\beta_{q}(X ; G)=\beta_{q}(Y ; G)+\beta_{q}(X \backslash Y ; G)
$$

for any equivariant inclusion $Y \subset X$ of $G$-orthogonal $\mathcal{A S}$-sets, proceeding once again by induction on the dimension (the property is obviously true for zero-dimensional $\mathcal{A} \mathcal{S}$-sets) : suppose the above equality to be true for any $G$-orthogonal $\mathcal{A S}$-sets of dimension $\leq d-1$.

First, if $T$ be a $d$-dimensional $G$-orthogonal $\mathcal{A S}$-set, then

$$
\beta_{q}(T ; G)=\beta_{q}\left(\bar{T}^{\mathcal{A S}} ; G\right)-\beta_{q}\left(\bar{T}^{\mathcal{A S}} \backslash T ; G\right) .
$$

Indeed, if $S:=\bar{T}^{\mathcal{A S}} \backslash T$, we have

$$
\beta_{q}(T ; G)=\beta_{q}\left(\bar{T}^{\mathcal{A S}} ; G\right)+\beta_{q}\left(\bar{S}^{\mathcal{A S}} \cap T ; G\right)-\beta_{q}\left(\bar{S}^{\mathcal{A S}} ; G\right)
$$

(see the proof of theorem 4.12). But $\bar{S}^{\mathcal{A S}} \cap T \subset \bar{S}^{\mathcal{A S}}$ is an equivariant inclusion of $G$ - $\mathcal{A S}$-sets of dimension $<d$ (we have $\operatorname{dim} S<\operatorname{dim} T$ and $\operatorname{dim} \bar{S}^{\mathcal{A S}}=\operatorname{dim} S:$ see [12] Proposition 3.3), so

$$
\beta_{q}\left(\bar{S}^{\mathcal{A S}} ; G\right)-\beta_{q}\left(\bar{S}^{\mathcal{A S}} \cap T ; G\right)=\beta_{q}\left(\bar{S}^{\mathcal{A S}} \backslash T ; G\right)=\beta_{q}\left(\bar{T}^{\mathcal{A S}} \backslash T ; G\right) .
$$

Now, consider an equivariant inclusion $Y \subset X$ of $G$-orthogonal $\mathcal{A S}$-sets with $\operatorname{dim} X=d$. If $X$ is compact, $\bar{Y}^{\mathcal{A S}} \subset X$ and $\bar{Y}^{\mathcal{A S}} \backslash Y \subset X \backslash Y$ are equivariant closed inclusions so that

$$
\begin{aligned}
\beta_{q}(X \backslash Y ; G) & =\beta_{q}\left(\bar{Y}^{\mathcal{A S}} \backslash Y ; G\right)+\beta_{q}\left(X \backslash \bar{Y}^{\mathcal{A S}} ; G\right) \\
& =\beta_{q}\left(\bar{Y}^{\mathcal{A S}} ; G\right)-\beta_{q}(Y ; G)+\beta_{q}(X ; G)-\beta_{q}\left(\bar{Y}^{\mathcal{A S}} ; G\right) \\
& =\beta_{q}(X ; G)-\beta_{q}(Y ; G)
\end{aligned}
$$

If $X$ is not compact, denote $X_{0}:=\bar{X}^{\mathcal{A S}} \backslash X$ and consider the equivariant closed inclusion ${\overline{X_{0}}}^{\mathcal{A S}} \cap(X \backslash Y) \subset X \backslash Y$. We have

$$
\beta_{q}(X \backslash Y ; G)=\beta_{q}\left({\overline{X_{0}}}^{\mathcal{A S}} \cap(X \backslash Y) ; G\right)+\beta_{q}\left((X \backslash Y) \backslash{\overline{X_{0}}}^{\mathcal{A S}} ; G\right) .
$$


Since $(X \backslash Y) \backslash{\overline{X_{0}}}^{\mathcal{A S}}=\left(\bar{X}^{\mathcal{A S}} \backslash Y\right) \backslash{\overline{X_{0}}}^{\mathcal{A S}} \subset \bar{X}^{\mathcal{A S}} \backslash Y$ is an equivariant open inclusion, we also have

$$
\beta_{q}\left((X \backslash Y) \backslash{\overline{X_{0}}}^{\mathcal{A S}} ; G\right)=\beta_{q}\left(\bar{X}^{\mathcal{A S}} \backslash Y ; G\right)-\beta_{q}\left({\overline{X_{0}}}^{\mathcal{A S}} \backslash Y ; G\right) .
$$

Finally, ${\overline{X_{0}}}^{\mathcal{A S}} \cap(X \backslash Y) \subset{\overline{X_{0}}}^{\mathcal{A S}} \backslash Y$ is an equivariant inclusion in dimension $<d$ so

$$
\beta_{q}\left({\overline{X_{0}}}^{\mathcal{A S}} \backslash Y ; G\right)-\beta_{q}\left({\overline{X_{0}}}^{\mathcal{A S}} \cap(X \backslash Y) ; G\right)=\beta_{q}\left(\bar{X}^{\mathcal{A S}} \backslash X ; G\right)
$$

and

$$
\begin{aligned}
\beta_{q}(X \backslash Y ; G) & =\beta_{q}\left(\bar{X}^{\mathcal{A S}} \backslash Y ; G\right)-\beta_{q}\left(\bar{X}^{\mathcal{A S}} \backslash X ; G\right) \\
& =\beta_{q}\left(\bar{X}^{\mathcal{A S}} ; G\right)-\beta_{q}(Y ; G)-\left(\beta_{q}\left(\bar{X}^{\mathcal{A S}} ; G\right)-\beta_{q}(X ; G)\right) \\
& =\beta_{q}(X ; G)-\beta_{q}(Y ; G) .
\end{aligned}
$$

To end the proof, we consider any equivariant inclusion $Y \subset X$ of $G$ - $\mathcal{A S}$-sets and we let $\varphi: X \rightarrow S$ to be a $G$-orthogonal embedding of $X$. Then $\varphi: Y \rightarrow \varphi(Y)$ is a $G$-orthogonal embedding of $Y$ and

$$
\beta_{q}(X ; G)=\beta_{q}(S ; G)=\beta_{q}(\varphi(Y) ; G)+\beta_{q}(\varphi(X \backslash Y) ; G)=\beta_{q}(Y ; G)+\beta_{q}(X \backslash Y ; G) .
$$

Let us then give the following definition :

Definition 4.15. Let e be a map from the category of $G-\mathcal{A S}$-sets and equivariant continuous $\mathcal{A S}$-maps to an abelian group $A$. We say that $e$ is an additive invariant of $G$-AS-sets if

- whenever $f: X \rightarrow X^{\prime}$ is an equivariant $\mathcal{A S}$-homeomorphism, then

$$
e(X)=e\left(X^{\prime}\right),
$$

- for any equivariant inclusion $Y \subset X$, we have

$$
e(X)=e(Y)+e(X \backslash Y) .
$$

The $G$-virtual Betti numbers are additive invariants of $G$ - $\mathcal{A S}$-sets. We define another one from them :

Definition 4.16. Let $X$ a $G$-AS-set. We set

$$
\beta(X ; G):=\sum_{q \in \mathbb{N}} \beta_{q}(X ; G) u^{q} \in \mathbb{Z}[[u]] .
$$

and we call this formal power series the G-virtual Poincaré series of $X$.

The $G$-virtual Poincaré series is an additive invariant of $G$-AS-sets, which coincides on compact nonsingular $G$-orthogonal $\mathcal{A S}$-sets with the generating function of the dimensions of the equivariant singular homology groups (we denote this generating function by $b(\cdot ; G)$ ). 
Remark 4.17. - If $T$ is any $G$-space and if we consider $H_{*}^{G}(T)$ as a graded $\mathbb{F}_{2}$-vector space, then $b(T ; G)=\sum_{q \in \mathbb{N}} \operatorname{dim}_{\mathbb{F}_{2}} H_{q}^{G}(T) u^{q}$ is called the Hilbert-Poincaré series of $H_{*}^{G}(T)$.

- The $G$-virtual Betti numbers and the $G$-virtual Poincaré series defined above are different from the equivariant virtual Betti numbers and equivariant virtual Poincaré series of [10] : they are not induced by the same equivariant homology. See also below example 4.18 (3).

- Let $q \in \mathbb{N}$. The map which associates to a $G$ - $\mathcal{A S}$-set $X$ the $q^{\text {th }}$ virtual Betti number (see [15], [8] and also [16]) of its fixed points set $\beta_{q}\left(X^{G}\right)$ is an additive invariant of $G$ - $\mathcal{A S}$-sets with values in $\mathbb{Z}$.

The virtual Poincaré polynomial of the fixed points set is also an additive invariant of $G$ - $\mathcal{A S}$-sets, with values in $\mathbb{Z}[u]$.

- If $G=\{e\}, \beta(\cdot ; G)$ is the virtual Poincaré polynomial of [8], since $H_{*}^{\{e\}}(T)=H_{*}(T)$ for any (compact) $G$-space (see remark $3.1(5)$ ).

Example 4.18. 1. Consider the $d$-dimensional affine space $\mathbb{A}:=\mathbb{R}^{d}$ equipped with any orthogonal action of a finite group $G$. In order to compute the equivariant virtual Poincaré series of $\mathbb{A}$, we consider the radial projection of $\mathbb{A}$ into the $d$-dimensional sphere $\mathbb{S}$ with center $\left(0, \ldots, 0, \frac{1}{2}\right)$ and radius $\frac{1}{2}$ of $\mathbb{R}^{d+1}$ : see for instance the proof of Proposition 3.5.12 of [4].

If we naturally extend the orthogonal action of $G$ into an orthogonal action on $\mathbb{R}^{d+1}$ (take the diagonal action fixing the last coordinate), this biregular embedding is equivariant (because the action preserves the euclidean norm). Denote by $p$ the point $(0, \ldots, 0,1)$ of $\mathbb{R}^{d+1}$. We then have

$$
\beta(\mathbb{A} ; G)=\beta(\mathbb{S} ; G)-\beta(p ; G)=b(\mathbb{S} ; G)-b(p ; G),
$$

since $\mathbb{S}$ and $p$ are compact and nonsingular.

We have $b(p ; G)=\sum_{q \in \mathbb{N}} \operatorname{dim}_{\mathbb{F}_{2}} H_{q}\left(G, \mathbb{F}_{2}\right) u^{q}$ (remark $\left.3.1(5)\right)$, and consider the $G-C W$ structure on $\mathbb{S}$ consisting in the $G$-invariant $d$-cell $\mathbb{A}$ and the $G$-invariant 0-cell $p$. Since all the cells are globally invariant under $G$, the action on the cellular complex $C_{*}^{\text {cell }}(\mathbb{S})$ is trivial and

$$
H_{*}(\mathbb{S} ; G)=H_{*}\left(G, C_{*}^{\text {cell }}(\mathbb{S})\right)=H_{*}(\mathbb{S}) \otimes_{\mathbb{F}_{2}} H_{*}\left(G, \mathbb{Z}_{2}\right)
$$

(see [6] VII-5 (5.4)), so that

$$
b(\mathbb{S} ; G)=b(\mathbb{S})\left(\sum_{q \in \mathbb{N}} \operatorname{dim}_{\mathbb{F}_{2}} H_{q}\left(G, \mathbb{F}_{2}\right) u^{q}\right)=\left(1+u^{d}\right)\left(\sum_{q \in \mathbb{N}} \operatorname{dim}_{\mathbb{F}_{2}} H_{q}\left(G, \mathbb{F}_{2}\right) u^{q}\right)
$$

$(b(\cdot)$ denotes the Poincaré polynomial). Finally

$$
\beta(\mathbb{A} ; G)=u^{d}\left(\sum_{q \in \mathbb{N}} \operatorname{dim}_{\mathbb{F}_{2}} H_{q}\left(G, \mathbb{F}_{2}\right) u^{q}\right)=u^{d} b(p ; G)
$$


2. We consider two different actions of $G:=\mathbb{Z} / 2 \mathbb{Z}$ on the hyperbola $X:=\{x y=1\}$ of $\mathbb{R}^{2}$.

First, consider the action given by the involution $\sigma:(x, y) \mapsto(-x,-y)$. Consider the projective Zariski closure $\bar{X}:=\left\{X Y=Z^{2}\right\}$ of $X$ in $\mathbb{P}^{2}(\mathbb{R})$, equipped with the involution $\bar{\sigma}:(X: Y: Z) \mapsto(-X:-Y: Z)=(X: Y:-Z)$. Then $X \subset \bar{X}$ is an equivariant inclusion (compactification) of $G$ - $\mathcal{A S}$-sets and $\bar{X} \backslash X=\{p, q\}$, where $p$ and $q$ are the points of respective homogeneous coordinates $[1: 0: 0]$ and $[0: 1: 0]$. Notice that $p$ and $q$ are fixed by the action of $G$ on $\bar{X}$ and that $\bar{X}$ is a nonsingular compact $G$ - $\mathcal{A S}$-set equipped with a biregular action of $G$ which is equivariantly homeomorphic to a circle equipped with a continuous action of $G$ with two fixed points. As a consequence,

$\beta(X ; G)=\beta(\bar{X} ; G)-\beta(\{p, q\} ; G)=(1+u) b(p ; G)-2 b(p ; G)=(u-1) \sum_{q \in \mathbb{N}} u^{q}=\frac{u-1}{1-u}=-1$ $\left(H_{k}\left(G, \mathbb{F}_{2}\right)=\mathbb{F}_{2}\right.$ if $k \geq 0$ for $G=\mathbb{Z} / 2 \mathbb{Z}:$ see for instance [10] Example 2.1).

If now we consider the action of $G$ given by the involution $\sigma:(x, y) \mapsto(y, x)$ (notice that the points of coordinates $(1,1)$ and $(-1,-1)$ are fixed by $\sigma)$, we equip $\bar{X}$ with the involution $\bar{\sigma}:(X: Y: Z) \mapsto(Y: X: Z)$, for which the two points $p$ and $q$ are exchanged. Consequently, $\beta(\{p, q\} ; G)=b(\{p, q\} / G)=b(p)=1($ see remark $3.1(5))$ and

$$
\beta(X ; G)=\beta(\bar{X} ; G)-\beta(\{p, q\} ; G)=(1+u) \sum_{q \in \mathbb{N}} u^{q}-1=\frac{2 u}{1-u} .
$$

3. Let $k$ and $l$ be odd integers and let $X$ be the real algebraic set $\left\{y^{2 l}=x^{2 k}\left(1-x^{2 k}\right)\right\}$ of $\mathbb{R}^{2}$. We will consider the actions of $G:=\mathbb{Z} / 2 \mathbb{Z}$ on $X$ given by the involutions $\sigma_{1}$ : $(x, y) \mapsto(-x, y), \sigma_{2}:(x, y) \mapsto(x,-y)$ and $\sigma_{3}:(x, y) \mapsto(-x,-y)$. First, notice that the map $(x, y) \mapsto\left(x^{k}, y^{l}\right)$ induces an equivariant homeomorphism with $\mathcal{A S}$-graph (actually algebraic graph) between the algebraic set $X^{\prime}:=\left\{y^{2}=x^{2}\left(1-x^{2}\right)\right\}$ of $\mathbb{R}^{2}$ and $X$, so that

$$
\beta(X ; G)=\beta\left(X^{\prime} ; G\right) .
$$

As in [10] Example 4.6, we consider an equivariant resolution of $X^{\prime}$ to compute the $G$ virtual Poincaré series of $X$. With the values of the $G$-virtual Poincaré series on the circle and points, we obtain

$$
\beta(X ; G)= \begin{cases}\frac{-u^{2}+u+1}{1-u} & \text { if } G=\left\{i d, \sigma_{1}\right\} \\ \frac{2 u+1}{1-u} & \text { if } G=\left\{i d, \sigma_{2}\right\} \\ \frac{u}{1-u} & \text { if } G=\left\{i d, \sigma_{3}\right\} .\end{cases}
$$

In particular, we see that the actions of the involutions $\sigma_{1}$ and $\sigma_{3}$ are different up to equivariant $\mathcal{A S}$-homeomorphism, which could not be proven using the equivariant virtual Poincaré series of [10] or the virtual Poincaré polynomial of the fixed points set.

Remark 4.19. In the previous examples, we wrote the $G$-virtual Poincaré series as rational fractions. We will see in below theorem 4.24 that, for any $G$ - $\mathcal{A S}$-set $X$, the $G$-virtual Poincaré series of $X$ can always be written as a rational fraction whose denominator depends only on the group $G$. So the information encoded by the $G$-virtual Poincaré series is actually finite and encoded in the numerator polynomial of this fraction. 


\subsection{The dual $G$-Nash constructible filtration and the finiteness of the $G$ - virtual Poincaré series}

We deal with the cohomological counterpart of the previous paragraphs, adapting the construction of the dual geometric filtration of [13], section 4, to our equivariant $\mathcal{A S}$ context. First, remark that $C^{q}(X ; G)=\operatorname{Hom}_{\mathbb{F}_{2}}\left(C_{q}(X ; G), \mathbb{F}_{2}\right)$, since

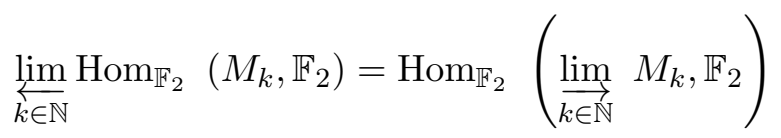

for any inductive system of $\mathbb{F}_{2}$-vector spaces $M_{k}, k \in \mathbb{N}$.

Definition 4.20. Let $X$ be a $G$-AS-set. For $p \in \mathbb{Z}, q \in \mathbb{N}$, we set

$$
\mathcal{N}^{p} C^{q}(X ; G):=\left\{\varphi \in C^{q}(X ; G) \mid \varphi \equiv 0 \text { on } \mathcal{N}_{p-1} C_{q}(X ; G)\right\} .
$$

This fits into a decreasing filtration $\mathcal{N}^{\bullet}$ on $C^{*}(X ; G)$ :

$$
0=\mathcal{N}^{1} C^{q}(X ; G) \subset \mathcal{N}^{0} C^{q}(X ; G) \subset \cdots \subset \mathcal{N}^{-q+1} C^{q}(X ; G) \subset \mathcal{N}^{-q} C^{q}(X ; G),
$$

that we call the dual $G$-Nash constructible filtration of $X$.

Since the filtration $\mathcal{N}^{\bullet}$ is bounded, it induces a spectral sequence $E_{*}^{*, *}(X ; G)$ which converges ([14] Theorem 2.6) to the cohomology of $C^{*}(X ; G)$, that is the equivariant cohomology with compact supports $H^{*}(X ; G)$ of $X$. The induced filtration

$$
0=\mathcal{N}^{1} H^{q}(X ; G) \subset \mathcal{N}^{0} H^{q}(X ; G) \subset \cdots \subset \mathcal{N}^{-q+1} H^{q}(X ; G) \subset \mathcal{N}^{-q} H^{q}(X ; G)
$$

on $H^{*}(X ; G)$ is called the cohomological $G$-weight filtration of $X$.

Just as in [13] section 4, we have natural isomorphisms

$$
\mathcal{N}^{p} C^{q}(X ; G)=\left(\frac{C_{q}(X ; G)}{\mathcal{N}_{p-1} C_{q}(X ; G)}\right)^{\vee}
$$

(where ${ }^{\vee}$ denotes the duality functor $\operatorname{Hom}_{\mathbb{F}_{2}}\left(\cdot, \mathbb{F}_{2}\right)$, which is exact), so that the cohomological $G$-weight spectral sequence is naturally dual to the (homological) $G$-weight spectral sequence :

$$
E_{r}^{p, q}(X ; G)=\left(E_{p, q}^{r}(X ; G)\right)^{\vee}
$$

for all $r \geq 0, p, q \in \mathbb{Z}$.

Moreover, the filtered cochain complex $\mathcal{N}^{\bullet} C^{*}(X ; G)$ is the inverse limit of the projective system $\mathcal{N}^{\bullet} C^{*}\left(X_{(k+1)}\right) \rightarrow \mathcal{N}^{\bullet} C^{*}\left(X_{(k)}\right)$ and the cohomological $G$-weight spectral sequence is the inverse limit of the induced cohomological weight spectral sequences (use the above natural isomorphisms (3), (2) and the exactness of the direct limit and duality functors).

Remark 4.21. The above cohomological $G$-weight filtration and spectral sequence are different from the ones of [23] section 3 . 
As in [13] Lemma 4.2, the additivity and acyclicity short exact sequences of theorem 4.4 and corollary 4.6 induces short exact sequences of additivity and acyclicity for the dual $G$-Nash constructible filtration. We deduce finite long exact sequences of additivity (and acyclicity) on the lines of the second page of the reindexed (take the same reindexation as in subsection $4.2)$ cohomological $G$-weight spectral sequence. We can therefore recover the $G$-virtual Betti numbers (theorem 4.12) from the cohomological $G$-weight spectral sequence as well :

Proposition 4.22. Let $X$ be $a G$-AS -set and let $q \in \mathbb{N}$. We have

$$
\beta_{q}(X ; G)=\sum_{p \in \mathbb{N}}(-1)^{p} \operatorname{dim}_{\mathbb{F}_{2}} \widetilde{E}_{2}^{p, q}(X ; G) .
$$

Proof. Each line of $\widetilde{E}_{2}(X ; G)$ is bounded because it is dual to its homological counterpart and because of theorem 4.11. Furthermore, thanks to the long exact sequence of additivity on each line of $\widetilde{E}_{2}^{p, q}(X ; G)$, the right member is additive. It is also an invariant of $G$ - $\mathcal{A S}$-sets with respect to equivariant $\mathcal{A S}$-homeomorphisms, because the dual $G$-Nash constructible filtration is a (contravariant) functor with respect to equivariant proper continuous $\mathcal{A S}$-maps, since so is the homological one (use again the natural isomorphisms (3)).

Finally, if $X$ is a compact nonsingular $G$-orthogonal $\mathcal{A S}$-set, we have

$$
\widetilde{E}_{p, q}^{2}(X ; G)= \begin{cases}H_{q}(X ; G)=H_{q}^{G}(X) & \text { if } p=0, \\ 0 & \text { if } p \neq 0,\end{cases}
$$

and

$$
\widetilde{E}_{2}^{p, q}(X ; G)=\left(\widetilde{E}_{p, q}^{2}(X ; G)\right)^{\vee}= \begin{cases}H^{q}(X ; G)=H_{G}^{q}(X) & \text { if } p=0, \\ 0 & \text { if } p \neq 0,\end{cases}
$$

so that $\sum_{p \in \mathbb{N}}(-1)^{p} \operatorname{dim}_{\mathbb{F}_{2}} \widetilde{E}_{2}^{p, q}(X ; G)=\operatorname{dim}_{\mathbb{F}_{2}} H_{q}^{G}(X)$.

We conclude by the uniqueness of the $q^{\text {th }} G$-virtual Betti number with these properties.

Remark 4.23. If $X$ is a compact nonsingular $G$-orthogonal $\mathcal{A S}$-set, we have a Poincaré duality isomorphism between $H^{*}(X ; G)$ and the equivariant homology considered in [10] (see [29] III Theorem 4.2, [10] 2.3.5 and also [23] Remark 4.26) so that

$$
\beta(X ; G)(u)=u^{d} \beta^{G}(X)\left(u^{-1}\right)
$$

where $d$ is the dimension of $X$ and $\beta^{G}(\cdot)$ is the equivariant virtual Poincaré series of [10].

However, this equality does not hold for general $G$ - $\mathcal{A S}$-sets. Consider for instance the third example of 4.18 : we have $\beta\left(X ;\left\{i d, \sigma_{1}\right\}\right) \neq \beta\left(X ;\left\{i d, \sigma_{3}\right\}\right)$ while $\beta^{\left\{i d, \sigma_{1}\right\}}(X)=\beta^{\left\{i d, \sigma_{3}\right\}}(X)$ (see [10] Example 4.6).

In particular, we notice that if $X$ is a compact nonsingular $G$-orthogonal $\mathcal{A S}$-set, $\beta(X ; G)$ equals the Hilbert-Poincaré series of the graded $\mathbb{F}_{2}$-vector space $H_{G}^{*}(X)$.

Now, remark that the equivariant singular cohomology has a richer algebraic structure than its homological counterpart. Namely, if $T$ is a finite-dimensional $G$-space, there is a cup 
product on $H_{G}^{*}(T)$, induced from the cup product in singular cohomology, which gives $H_{G}^{*}(T)$ a structure of graded $\mathbb{F}_{2}$-algebra.

If $p$ is a point, the map $T \rightarrow\{p\}$ induces, by contravariant functoriality, a morphism of graded $\mathbb{F}_{2}$-algebras $H^{G}(\{p t\})=H^{*}\left(G, \mathbb{F}_{2}\right) \rightarrow H_{G}^{*}(T)$, which gives $H_{G}^{*}(T)$ a structure of graded module over $H^{*}\left(G, \mathbb{F}_{2}\right)$. But, since $G$ is a finite group, $H^{*}\left(G, \mathbb{F}_{2}\right)$ is a finitely generated graded $\mathbb{F}_{2}$-algebra and, since $H^{*}(T)$ is a finitely generated graded module over $\mathbb{F}_{2}, H_{G}^{*}(T)$ is a finitely generated graded module over $H^{*}\left(G, \mathbb{F}_{2}\right)$ (see [25] Theorem 2.1 and Corollaries 2.2 and 2.3).

As a consequence, if $x_{1}, \ldots, x_{k}$ are homogeneous generators of the graded $\mathbb{F}_{2}$-algebra $H^{*}\left(G, \mathbb{F}_{2}\right)$, with respective degrees $d_{1}, \ldots, d_{k}$ in the graduation, by the Hilbert-Serre theorem (see for instance [2] Theorem 11.1), there exists a polynomial $f(u) \in \mathbb{Z}[u]$ such that the Hilbert-Poincaré series of $H_{G}^{*}(T)$ can be written

$$
b(T ; G)=\frac{f(u)}{\prod_{i=1}^{m}\left(1-u^{d_{i}}\right)} .
$$

We deduce a similar form for the $G$-virtual Poincaré series :

Theorem 4.24. Let $X$ be a $G$-AS-set. There exists a polynomial $F(u) \in \mathbb{Z}[u]$ such that

$$
\beta(X ; G)=\frac{F(u)}{\prod_{i=1}^{m}\left(1-u^{d_{i}}\right)} .
$$

Proof. Proceed as in the proof of theorem 4.12 : prove the result on $G$-orthogonal $\mathcal{A S}$-sets using induction on dimension and the fact that the property is true on compact nonsingular $G$-orthogonal $\mathcal{A S}$-sets, together with the fact that the sum of two fractions of the above form is again of this form.

Example 4.25. If $G=\mathbb{Z} / 2 \mathbb{Z}$, then $H^{*}\left(G, \mathbb{F}_{2}\right)$ is isomorphic to $\mathbb{F}_{2}[u]$ as a graded $\mathbb{F}_{2}$-algebra, with $u$ of degree 1 , so that, if $X$ is any $G$ - $\mathcal{A S}$-set,

$$
\beta(X ; G)=\frac{F(u)}{1-u}
$$

with $F(u) \in \mathbb{Z}[u]$.

Remark 4.26. If, for a $G$ - $\mathcal{A S}$-set $X$, we denote $\mathfrak{b}(X ; G):=\prod_{i=1}^{m}\left(1-u^{d_{i}}\right) \beta(X ; G) \in \mathbb{Z}[u]$, the map $X \mapsto \mathfrak{b}(X ; G)$ is a polynomial additive invariant of $G$ - $\mathcal{A} \mathcal{S}$-sets. Notice that this invariant depends on the considered generators of the graded $\mathbb{F}_{2}$-algebra $H^{*}\left(G, \mathbb{F}_{2}\right)$.

\section{Other properties of the $G$-virtual Poincaré series and appli- cations}

In this final section, we show that the $G$-virtual Poincaré series has properties similar to the ones of the equivariant virtual Poincaré series of [10], which allows it to be used to define helpful tools (namely zeta functions) for the classification of real analytic germs.

All the story begins with the following property, similar to [10] Proposition 3.13 : 
Proposition 5.1. Let $X$ be a $G$-AS-set and let $\mathbb{R}^{d}$ be an affine space equipped with any orthogonal action of $G$. Then

$$
\beta\left(X \times \mathbb{R}^{d} ; G\right)=u^{d} \beta(X ; G)
$$

(on the left-hand side, we consider the diagonal action of $G$ on $X \times \mathbb{R}^{d}$ ).

Before proving the above result, we need to mention the following facts. First, remark that, if $G$ and $H$ are finite groups and if $X$ and $Y$ are respectively a $G$-space and an $H$-space, we have a Künneth-type formula

$$
H_{*}^{G}(X) \otimes_{\mathbb{Z}_{2}} H_{*}^{H}(Y) \cong H_{*}^{G \times H}(X \times Y) .
$$

Indeed, if $E_{G}$, resp. $E_{H}$, is a contractible topological space equipped with a free action of $G$, resp. $H$, then $E_{G} \times E_{H}$ is a contractible space with a free action of $G \times H$ and $(X \times Y) \times_{G \times H}\left(E_{G} \times E_{H}\right)$ is naturally isomorphic to $\left(X \times_{G} E_{G}\right) \times\left(Y \times_{H} E_{H}\right)$, so that the usual Künneth formula for homology can be applied.

We will use this property together with the following one : suppose that $X$ and $Y$ are two $G$ - $C W$-complexes such that the action $G$ globally stabilizes each cell of $Y$, then $H_{*}^{G}(X \times Y)=$ $H_{*}^{G \times\{e\}}(X \times Y)$, where, on the left-hand side, $G$ acts diagonally on $X \times Y$ and, on the right-hand side, we make the trivial group act on $Y$.

Indeed, compute the equivariant homology of $X \times Y$ as the cellular homology of the quotient of $(X \times Y) \times E_{G}$ by $G$. Take $E_{G}$ to be a $G-C W$-complex such that its cells are freely permuted by $G$ ([11] Example 1B.7) : a cell of the quotient is then the orbit of freely permuted cells of $(X \times Y) \times E_{G}$ (which are the products of the cells of $X, Y$ and $\left.E_{G}\right)$ under the action of $G$. Since the actions of $G$ and $G \times\{e\}$ on the cells of $(X \times Y) \times E_{G}$ have the same orbits, we get the result.

Proof of proposition 5.1. We can suppose $X$ to be a $G$-orthogonal $\mathcal{A S}$-set. The proof is then analogous to the proof of Proposition 3.13 of [10] : first, suppose that $X$ is a compact and nonsingular $G$-orthogonal $\mathcal{A S}$-set and equivariantly compactify $\mathbb{R}^{d}$ into the $d$-dimensional sphere $\mathbb{S}^{d}$ as in above example $4.18(1): X \times \mathbb{R}^{d} \hookrightarrow X \times \mathbb{S}^{d}$ is then an equivariant compactification of $X \times \mathbb{R}^{d}$.

Consider a $G$-CW-structure on $X([19])$ and the $G$-CW-structure on $\mathbb{S}^{d}$ consisting in the $G$-invariant $d$-cell $\mathbb{R}^{d}$ and the $G$-invariant 0 -cell $\{p\}:=\mathbb{S}^{d} \backslash \mathbb{R}^{d}$. Since $X$ and $\mathbb{S}^{d}$ are compact and nonsingular, we have

$\beta\left(X \times \mathbb{S}^{d} ; G\right)=b\left(X \times \mathbb{S}^{d} ; G\right)=b\left(X \times \mathbb{S}^{d} ; G \times\{e\}\right)=b(X ; G) \cdot b\left(\mathbb{S}^{d} ;\{e\}\right)=b(X ; G) \cdot b\left(\mathbb{S}^{d}\right)=\left(1+u u^{d}\right) b(X ; G)$,

and, by additivity of the $G$-virtual Poincaré series,

$\beta\left(X \times \mathbb{R}^{d} ; G\right)=\beta\left(X \times \mathbb{S}^{d} ; G\right)-\beta(X \times\{p\} ; G)=\left(1+u^{d}\right) b(X ; G)-b(X ; G)=u^{d} b(X ; G)=u^{d} \beta(X ; G)$.

The rest of the proof proceeds just as in [10], using an induction on the dimension of $X$ and the additivity of the $G$-virtual Poincaré series $\beta(\cdot ; G)$ (see also the proofs of theorems 4.11 and 4.12). 
Remark 5.2. The equality (4) remains true for any $G$ - $\mathcal{A S}$-action on the affine space $\mathbb{R}^{d}$ such that $\mathbb{R}^{d}$ can be equivariantly compactified into a $d$-dimensional sphere via an equivariant $\mathcal{A S}$ homeomorphism.

Thanks to proposition 5.1, the $G$-virtual Poincaré series could be used to define invariants, in terms of (motivic) zeta functions, for some equivalence relation of equivariant Nash germs, namely equivariant blow-Nash equivalence (see [9]) or equivariant arc-analytic equivalence (see [7]), just as as in [21]. Indeed, this is this key property of the equivariant virtual Poincaré series of [10], together with its additivity, which allow to prove Propositions 3.14 and 3.17 of [21].

This could be applied to study the classification of simple Nash germs invariant under the involution changing the sign of the first coordinate, as in [22].

We finally state the analogs of Proposition 3.14 and 3.15 of [10] for the $G$-virtual Poincaré series :

Proposition 5.3. Let $X$ be a $G$ - $\mathcal{A S}$-set.

1. If the action of $G$ on $X$ is trivial, then $\beta(X ; G)=\beta(X)\left(\sum_{q \in \mathbb{N}} H_{q}\left(G, \mathbb{F}_{2}\right) u^{q}\right)$.

2. If $X$ is a free $G$-AS-set, then the quotient $X / G$ is well-defined as an $\mathcal{A S}$-set (proposition 2.29) and $\beta(X ; G)=\beta(X / G)$.

Proof. For the first point, proceed just as in the proof of Proposition 3.14 of [10], considering $G$-orthogonal embedding and using an induction on dimension, as well as the Kunneth isomorphism $H_{*}(X ; G)=H_{*}(X) \otimes_{\mathbb{Z}_{2}} H_{*}\left(G, \mathbb{Z}_{2}\right)$ (see remark $3.1(5)$ ) when $X$ is a compact and nonsingular $G$-orthogonal $\mathcal{A S}$-set.

For the second point, consider a free $G$-orthogonal embedding and use also an induction on dimension. For the compact case, proceed as in the proof of Proposition 3.15 of [10], considering an equivariant resolution of the singularities of (the free $G$-orthogonal embedding of) $X$. If $X$ is not compact, apply the previous case to $\bar{X}^{\mathcal{A S}}$ (the action of $G$ on $\bar{X}^{\mathcal{A S}}$ is free by definition) and the induction hypothesis to $\bar{X}^{\mathcal{A S}} \backslash X$ to obtain, by additivity of the $G$-virtual Poincaré series,

$$
\beta(X ; G)=\beta\left(\bar{X}^{\mathcal{A S}} ; G\right)-\beta\left(\bar{X}^{\mathcal{A S}} \backslash X ; G\right)=\beta\left(\bar{X}^{\mathcal{A S}} / G\right)-\beta\left(\left(\bar{X}^{\mathcal{A S}} \backslash X\right) / G\right)=\beta(X / G) .
$$

\section{References}

[1] S. Akbulut, H. King, The Topology of Real Algebraic Sets, MSRI Publications no 25, Springer-Verlag, New York, 1992.

[2] M. F. Atiyah, I. G. Macdonald, Introduction to Commutative Algebra, Addison-Wesley, 1969 .

[3] E. Bierstone and P.D. Milman, Canonical desingularization in characteristic zero by blowing up the maximum strata of a local invariant, Invent. Math. 128 (1997), pp 207-302. 
[4] J. Bochnak, M. Coste, M.-F. Roy, Real Algebraic Geometry, Springer-Verlag, New York, 1992.

[5] A. Borel, Seminar on Transformation Groups, Annals of Mathematics Studies no 46, Princeton University Press, Princeton, 1960.

[6] K.S. Brown, Cohomology of groups, Graduate texts in Mathematics, 87, Springer-Verlag, 1982.

[7] J.-B. Campesato, On a motivic invariant of the arc-analytic equivalence, Ann. Inst. Fourier (Grenoble), 67 (2017), pp. 143-196.

[8] G. Fichou, Motivic invariants of Arc-symmetric sets and Blow-Nash equivalence, Compositio Math., 141, 2005, pp 655-688.

[9] G. Fichou, Zeta functions and Blow-Nash equivalence, Ann. Polon. Math. 87 (2005), pp 111-125.

[10] G. Fichou, Equivariant virtual Betti numbers, Ann. de l'Inst. Fourier, 58, no. 1 (2008), $1-27$.

[11] A. Hatcher, Algebraic Topology, Cambridge University Press, 2002.

[12] K. Kurdyka, A. Parusiński, Arc-symmetric sets and arc-analytic mappings, Panoramas et Synthèses 24, Soc. Math. France (2007), pp 33-67.

[13] T. Limoges, F. Priziac, Cohomology and products of real weight filtrations, Annales de l'Institut Fourier, 2015, 65 (5), pp.2235-2271, doi:10.5802/aif.2987

[14] J. McCleary, A User's Guide to Spectral Sequences (second edition), Cambridge University Press, 2001.

[15] C. McCrory, A. Parusiński, Virtual Betti numbers of real algebraic varieties, C. R. Math. Acad. Sci. Paris 336 (2003), no 9, pp 763-768.

[16] C. McCrory, A. Parusiński, The weight filtration for real algebraic varieties, Topology of stratified spaces, 121-160, Math. Sci. Res. Inst. Publ. 58, Cambridge Univ. Press, Cambridge, 2011.

[17] E. Noether, Der Endlichkeitssatz der Invarianten endlicher Gruppen, Mathematische Annalen, 77 no 1 (1915), pp 89-92.

[18] Y. Ozan, Quotients of real algebraic sets via finite group actions, Tr. J. of Mathematics, 21, 1997, pp 493-499.

[19] D. H. Park, D. Y. Suh, Semialgebraic G CW complex structure of semialgebraic G spaces, J. Korean Math. Soc., 35 no 2 (1998), pp 371-386. 
[20] F. Priziac, Equivariant weight filtration for real algebraic varieties with action, Journal of the Mathematical Society of Japan, 2016, 68 (4), pp 1789-1818, doi :10.2969/jmsj/06841789

[21] F. Priziac, Equivariant zeta functions for invariant Nash germs, Nagoya Mathematical Journal, 222 (1), 2016, pp 100-136, doi: 10.1017/nmj.2016.12

[22] F. Priziac, On the equivariant blow-Nash classification of simple invariant Nash germs, preprint, arXiv:1606.00303, submitted, 2016.

[23] F. Priziac, Products of real equivariant weight filtrations, preprint, arXiv:1708.05175, submitted, 2017.

[24] C. Procesi, G. Schwarz, Inequalities defining orbit spaces, Invent. Math., 81, 1985, pp 539-554.

[25] D. Quillen, The spectrum of an equivariant cohomology ring: I, Ann. of Math., 94, 1971, pp 549-572.

[26] G. Schwarz, Smooth functions invariant under the action of a compact Lie group, Topology, 14, 1975, pp 63-68.

[27] I. R. Shafarevich, Basic Algebraic Geometry 1, Springer-Verlag, Berlin, 1994.

[28] E. Spanier, Algebraic Topology, Springer-Verlag, New York 1966.

[29] J. van Hamel, Algebraic cycles and topology of real algebraic varieties, CWI Tract 129, Stichting Mathematisch Centrum, Centrum voor Wiskunde en Informatica, Amsterdam, 1997. 